Research Article

\title{
SufB intein splicing in Mycobacterium tuberculosis is influenced by two remote conserved $\mathrm{N}$-extein Histidines
}

Running Title: Conserved histidines regulate Mtu SufB cleavage

Sunita Panda ${ }^{1 \dagger}$, Ananya Nanda ${ }^{1 \dagger}$, Nilanjan Sahu ${ }^{2}$, Deepak Ojha ${ }^{1}$, Biswaranjan Pradhan ${ }^{3}$, Anjali Rai $^{1}$, Amol R. Suryawanshi ${ }^{4}$, Nilesh Banavali ${ }^{5,6}$ and Sasmita Nayak ${ }^{1 *}$

${ }^{1}$ School of Biotechnology, Kalinga Institute of Industrial Technology, Bhubaneswar, Odisha, India 751024 ,

${ }^{2}$ School of Biological Sciences, National Institute of Science Education and Research Bhubaneswar, Jatni, Khurda, Odisha, India 752050

${ }^{3}$ S K Dash center of excellence of Biosciences and Engineering \& Technology, School of Basic Sciences, Indian Institute of Technology Bhubaneswar, Argul, Khurda, Odisha, India 752050

${ }^{4}$ Clinical Proteomics, Institute of Life Sciences, Bhubaneswar, Odisha 751023 India

${ }^{5}$ Laboratory of Cellular and Molecular Basis of Diseases, Division of Transitional Medicine, Wadsworth Center, New York State Department of Health, Empire State Plaza, Albany New York 12237

${ }^{6}$ Department of Biomedical Sciences, School of Public Health, 1 University Place, Rensselaer, New York 12144

${ }^{\dagger}$ These authors contributed equally: Sunita Panda, Ananya Nanda.

*Correspondence: Dr Sasmita Nayak, School of Biotechnology, Kalinga Institute of Industrial Technology, Campus XI, Patia, Bhubaneswar, Odisha, India 751024

Email:-sasmita.n@kiitbiotech.ac.in; Tel: +918763000005

\section{Abbreviations:}

Mtu: Mycobacterium tuberculosis

WT: Wild type

SI: Splicing inactive

DM: Mtu SufB Double mutant 
IPTG: Isopropyl $\beta$ - d-1-thiogalactopyranoside

TCEP: Tris (2-carboxyethyl) phosphine

HA: Hydroxylamine

DTT: Dithiothreitol

HRP: Horseradish peroxidase

ECL: Enhanced chemiluminescence

TBST: Tris-buffered saline, $0.1 \%$ tween 20

BSA: Bovine serum albumin

B-gal: Beta galactosidase

ABC: Ammonium bicarbonate

ACN: Acetonitrile

TFA: Tri-fluoro acetic acid

HPLC: High pressure (or performance) liquid chromatography

FPLC: First protein liquid chromatography

MALDI-TOF/TOF: Matrix assisted laser desorption ionization time-of-flight

SDS: Sodium dodecyl sulphate

PAGE: Polyacrylamide gel electrophoresis

RMSD: Root mean square deviation

SASA: Solvent accessible surface area

RMSF: Root mean square fluctuation

PME: Particle-mesh Ewald

HPCC: High performance computing cluster

MD Simulations: Molecular dynamic simulations 
Abstract: Inteins are auto-processing domains that implement a multi-step biochemical reaction termed protein splicing, marked by cleavage and formation of peptide bonds. They excise from a precursor protein, generating a functional protein via covalent bonding of flanking exteins. We report the kinetic study of splicing and cleavage reaction in [Fe-S] cluster assembly protein SufB from Mycobacterium tuberculosis. Although it follows a canonical intein splicing pathway, distinct features are added by extein residues present in the active site. Sequence analysis identified two conserved histidines in the N-extein region; His-5 and His-38. Kinetic analyses of His-5Ala and His-38Ala SufB mutants exhibited significant reductions in splicing and cleavage rates relative to the SufB wild-type precursor protein. Structural analysis and molecular dynamics simulations suggested that Mtu SufB displays a unique mechanism where two remote histidines work concurrently to facilitate $\mathrm{N}$ - terminal cleavage reaction. His-38 is stabilized by the solvent-exposed His-5, and can impact N-S acyl shift by direct interaction with the catalytic Cys1. Development of inteins as biotechnological tools or as pathogen specific novel antimicrobial targets requires a more complete understanding of such unexpected roles of conserved extein residues in protein splicing.

Keywords: Intein splicing; Splicing regulation; conserved histidines; Mycobacterium tuberculosis SufB; SufB intein.

\section{Introduction}

Protein splicing is a self-catalyzed event that generates a continuous extein protein by ligating two intein separated extein regions with a peptide bond. This post-translational auto-excision of the intervening intein protein is critical for the formation of an active protein (1-4). Splicing and cleavage products (Figure 1C) are obtained through a series of nucleophilic displacement reactions mediated in a coordinated fashion by the catalytic residues (5-9). Control over this 
76 reversible interruption of the functional form of the host exteins can play a regulatory role in

protein activation $(10,11)$. The extein sequences upstream and downstream of N-terminal and Cterminal intein ends are termed 'N-extein' and 'C-extein' respectively. The intein folds into a horseshoe-shaped structure with a catalytic cleft that brings the conserved catalytic residues and the extein splice junction close enough for initiation of splicing reaction (12).

Inteins themselves have $\mathrm{N}$-terminal and $\mathrm{C}$-terminal regions with conserved sequence segments (termed as Blocks or motifs) that facilitates the splicing and cleavage reaction(s). N-terminal intein region comprises of A, N2, B, and N4 structural motifs or Blocks while F-and G-Blocks are part of the C-terminal intein region. Typically, A-Block contains Cys/Ser or Thr; B-Block includes His and Thr residues; F-Block usually has Asp and His and the G-Block bears two conserved residues; a penultimate His and a terminal Asn. Classical or Canonical (Class 1) intein splicing involves 4 sequential acyl rearrangements (Figure 1C), a nucleophilic attack by $\mathrm{C} 1$ or S1/T1 leads to an N-S/N-O acyl shift converting the peptide bond of N-terminal splice junction to a thioester linkage, a second nucleophilic attack by $\mathrm{C}+1$ forms a branched intermediate at $\mathrm{C}$ terminal splice junction through esterification, the branched intermediate is resolved by terminal Asn cyclization through cleavage of C-terminal splice junction, and finally an (S-N/O-N) acyl shift fully ligates the two extein segments by an amide bond formation.

These bond rearrangements at splice junctions during the cleavage and splicing reaction(s) are assisted by non-catalytic intein residues through stabilization of various intermediate structure(s)(13). The Block A residues Cys, Ser, or Thr participate in the first step of splicing with significant assistance from the Block B His and Thr residues. The highly conserved Block B His destabilizes the scissile peptide bond either by reducing the energy barrier or by loss of resonance or protonation of the Cys1 amide bond via His imidazole ring to catalyze the N-S acyl 
shift (14-18).The Block F Asp residue drives the thioesterification through the tetrahedral intermediate by ground-state destabilization $(8,16,19)$. It is also proposed that Block B histidine plays a dual catalytic role; being weakly basic it deprotonates the Cys1 to accelerate the N-S acyl shift and subsequently acts as an acid to stabilize the tetrahedral intermediate $(15,17,18)$. The Block F Asp residue also deprotonates the $\mathrm{C}+1$ residue to stabilizes the net positive charge on Cys1 that drives the transesterification reaction. The F- and G-Block His residues are critical in the coordination of terminal Asn cyclization. The F-Block His increases nucleophilicity of Asn, and the G-Block His accelerates the Asn cyclization by increasing the electrophilicity of backbone peptide (20-23). The final acyl-shift is energetically favorable and does not require assistance from either intein or extein residues(24). Inteins do show polymorphisms in the catalytic residues leading to variation in the splicing mechanism as seen in Class 2 and Class 3 intein splicing $(11,25-29)$.

Interactions between extein residues and the catalytic intein core in the regulation of splicing reaction have been studied by modulating intein activity, changing the conformation of catalytic cleft, and restraining the activity of catalytic residues $(6,30-34)$. Previous studies on intein-extein partnership in intein splicing have suggested mediation by extein residues both near and remote to the $\mathrm{N}$ - and $\mathrm{C}$-terminal splice junctions $(7,30,31,33,35-37)$. The $\mathrm{N}$-extein residue at the first position (-1) is important for the first thioester reaction and shows enhanced $\mathrm{N}$-terminal cleavage rate ( $>4$-fold) or attenuated cleavage by 1,000-fold by replacing the native residue to aspartate and proline respectively (30). Substitution of bulky amino acids at this position can cause local distortion and induce N-cleavage reaction (17). The participation of the first C-extein Cys +1 in the second and third steps of the splicing reaction has been demonstrated by its mutation dramatically augmenting or inhibiting splicing and generating off-pathway N-cleavage products 
$(12,35,38-40)$. Earlier, extein effects were assumed to be limited to residues proximal to the intein $(12,31,41)$, but recent work has shown that distal exteins are implicated as environmental sensors with the role in regulating splicing depending on solution environment and temperature in Pho RadA precursors $(6,10,34)$.

SufB is a critical component of [Fe-S] cluster assembly and repair machinery called SUF (mobilization of sulfur) complex. This is a stress response system that gets upregulated during periods of oxidative stress and Fe starvation $(42,43)$. Though there are multiple pathways for [Fe-S] cluster biogenesis among the three kingdoms of life such as NIF (nitrogen fixation) and ISC (Iron-sulfur cluster), the SUF complex is unique in mycobacteria. Fe-S cluster containing proteins execute a broad spectrum of cellular functions in organisms such as respiration, gene regulation, RNA modification, DNA repair, and replication (44-46). Although the SUF system has been well characterized in the E. coli system, $(39,42,43,47-49)$, the importance of SufB intein splicing in the formation of functional Suf complex has been shown in mycobacteria (49).

The present study reports on the splicing and cleavage reactions of a full-length Mtu SufB (FLSufB) precursor protein. We delineated the different structural domains of Mtu SufB, analyzed whether it follows a canonical or non-canonical intein splicing pathway, identified intein and extein residues that participate in catalytic cleft formation, assessed both their conservation in different mycobacterial species and their role in regulating cleavage and/or splicing reactions, and analyzed distinctions from other intein precursor proteins. We found intein residues highly conserved in different mycobacterial species that favor a canonical splicing mechanism (Figure $1 \mathrm{~A}$ and 1B). We detected two distal histidines in the N-extein region, His-5 and His-38, that are conserved in mycobacteria, archaea, and other microbes where SUF is the exclusive system for Fe-S cluster biogenesis (Figure 2A and 2B). Biochemical analyses of H5A and H38A SufB 
mutants confirmed their influence on splicing and cleavage. Structural modeling of Mtu SufB and explicit-solvent molecular dynamics (MD) simulations of the model were used to analyze the SufB precursor splicing active site dynamics, and these simulations suggested that $\mathrm{N}$-terminal cleavage could be supported by an interaction between H- 38 and H-5. These observations suggest that the two distal H-5 and H-38 N-extein residues participate in SufB precursor stabilization and aid Mtu SufB intein splicing.

Kinetic analyses of H-5A SufB mutant demonstrated 3-fold and 1.4-fold reductions in splicing and cleavage rates respectively, relative to wild-type Mtu SufB precursor. Likewise, a 3.4-fold and 3-fold reduction in splicing and cleavage rates were observed in the H-38A SufB mutant. A side-by-side Mtu SufB structure prediction was done using homology (chimera) modeling, secondary structure prediction through consensus with other protein sequences. Subsequently, molecular dynamics (MD) simulations in aqueous media were carried out to find the equilibrated structure. Furthermore, MD simulations clarified the structural features of the SufB intein active site and indicated that $\mathrm{N}$-terminal cleavage reaction is catalyzed by $\mathrm{H}-38$ with the assistance of H-5. Taken together, our study substantiates a distinct mechanism for $\mathrm{N}$-terminal cleavage reaction shown by $M t u$ SufB. Although H-38 is relatively distal to the N-terminal splice junction when supported by His-5, it can efficiently activate the first step of splicing reaction. Finally, we have proposed a novel mechanism for the $\mathrm{N}$-cleavage reaction mediated via the concerted actions of these conserved histidines in the N-extein region of Mtu FL-SufB precursor. Interestingly, attempts to express the SufB double mutant $(\mathrm{H}-5 \mathrm{~A} / \mathrm{H}-38 \mathrm{~A})$ protein resulted in a truncated protein. These observations suggest that H-5 and H-38 might have important biological role(s) in the SufB precursor stabilization and perhaps the functionality of Mtu SufB protein. 


\section{MATERIALS AND METHODS}

\subsection{Genetic constructs}

The full-length Fe-S cluster assembly protein SufB from the Mycobacterium tuberculosis H37Rv strain (Mtu-SufB-FL) and its isolated intein (Mtu-SufB-I) genes were PCR-amplified using Pfu Ultra High-Fidelity DNA Polymerase (Agilent Technologies) from heat-killed Mtu genomic DNA. DNA purification by gel electrophoresis was followed by EcoRI and HindIII restriction digestion and cohesive end ligation (T4 DNA Ligase, NEB Cat. No. M0202S) for cloning. The genes were inserted into the multiple cloning site 1 (MCS1) of the low copy expression vector pACYCDuetTM-1(Novagen), which was driven by a T7 promoter/lac operator with a chloramphenicol resistance gene for selection. The constructs were screened via colony PCR and confirmed by sequencing (Sequencing Core Facility, SUNY, Albany, and Eaton Bioscience Inc. sequencing service) using the ACYCDuetUP1 (Novagen Cat. No.71178-3) and DuetDOWN1 primers (Novagen Cat. No. 71179-3), as well as the original primers. Mtu-SufB-FL mutants H5A, H38A, C1A, N359A were generated by substituting respective key catalytic residues to alanine via phosphorylated inverse PCR primers. Splicing inactive (SI) Mtu-SufB-FL double mutant (C1A/N359A) was created via inverse PCR to add the N359A mutation into the C1A cleavage mutant. Similarly, H5A/H38A Mtu-SufB-FL double mutant (DM) was created by adding H38A mutation into the H5A mutant by inverse PCR. The cloned H38A and H5A/H38A Mtu SufB mutant genes were also confirmed separately by sequencing (Agrogenomics, Odisha) using ACYCDuetUP1 (IDT Cat. No. 103948189) and DuetDOWN1 (IDT Cat. No. 103948190) along with the SufB Primers. All the above primers are listed in Table S1. 


\subsection{Sequence analysis}

Protein sequences for Mtu SufB-FL (Accession number YP_006514844.1, GI: 397673309) and the 477 amino acid intein-less SufB protein from Mycobacterium smegmatis strain MC2 155 (Msm-SufB-FL, accession number YP_887437.1, GI: 118472504) were pairwise sequence aligned in ClustalW to distinguish extein and intein regions in Mtu SufB-FL (50). The identified Mtu SufB intein sequence (Mtu SufB-I) was confirmed by sequence comparison using Blast with the sequences deposited in Inbase (The Intein Database, www.inteins.com) (51). Different structural domains of the intein-like homing endonuclease, and the $\mathrm{N}$ - and $\mathrm{C}$-terminal inteins were clearly demarcated by sequence alignment and structural analysis of SufB intein with homing endonuclease domain (I-CreI) and intein homing endonuclease Ii (PDB: 2CW7). The Mtu SufBFL and $M s m$-SufB-FL sequences, combined with one archaeal and other bacterial SufB proteins collected from the NCBI and intein databases (51), were aligned using Dialign2 software (52). Conservation of different intein and extein residues was edited and color-coded manually. Phylogenetic tree analysis was performed using the Maximum likelihood method in the MEGA X program $(53,54)$ for both SufB inteins [Figure 2C (ii)] and SufB precursor sequences [Figure 2C (i)] from different organisms.

\subsection{Protein overexpression and purification}

Full-length (FL) un-spliced precursor and mutant SufB proteins carrying an N-terminal 6X-Histag were over-expressed in BL21 (DE3) E.coli cells via IPTG (Sigma 367-93-1) induction. Cells were resuspended in lysis buffer $(20 \mathrm{mM}$ sodium phosphate, $0.5 \mathrm{M} \mathrm{NaCl}, \mathrm{pH} 7.4)$ and lysed via tip sonicator (Sonics vibra cell VCX-130). Proteins were over-expressed and isolated from inclusion bodies (IB) via centrifugation. The IB materials were solubilized by $8 \mathrm{M}$ urea (Merck, 1084870500) 
buffer (lysis buffer, 8M urea, $20 \mathrm{mM}$ of imidazole (MP-biochemicals-288-32-4) and centrifuged at $16,500 \mathrm{~g}$ for $20 \mathrm{~min}$ to collect the supernatant. Then $6 \mathrm{X}$-His-tagged full-length (FL) precursor and mutant proteins were purified by Ni-NTA affinity column (Ni-NTA His trap, HP GE Healthcare Life Sciences-17524802). Prior to sample application, columns were equilibrated with binding buffer (20mM sodium phosphate, $0.5 \mathrm{M} \mathrm{NaCl}, 40 \mathrm{mM}$ imidazole). After sample loading, columns were washed several times $(15 \mathrm{CV})$ in binding buffer. Finally, proteins were eluted as purified fractions in elution buffer (20mM sodium phosphate, $0.5 \mathrm{M} \mathrm{NaCl}, 500 \mathrm{mM}$ imidazole) followed by quantification via Bradford's assay.

\subsection{In-vitro splicing and cleavage assays}

$2.5 \mu \mathrm{M}$ of purified proteins were allowed to refold in $1 \mathrm{ml}$ of renaturation buffer ( $20 \mathrm{mM}$ sodium phosphate, $0.5 \mathrm{M} \mathrm{NaCl}, 0.5 \mathrm{M}$ Arginine, $1 \mathrm{mM}$ EDTA, $\mathrm{pH}$ 7.4) in presence of $2 \mathrm{mM}$ TCEP-HCl (sigma-51805-45-9) at $20^{\circ} \mathrm{C}$ for $24 \mathrm{~h}$. The $0 \mathrm{hr}$ sample was retrieved before renaturation and splicing was quenched by addition of loading dye $(0.1 \%$ bromophenol blue, $50 \%$ glycerol, $\beta$ mercaptoethanol, $10 \%$ SDS, tris 6.8 ) followed by rapid freezing at -200 C. Our controls, splicing inactive(SI) SufB double mutant and empty expression vector pACYC Duet-1, were treated similarly for the in-vitro assays. For the N-cleavage assay, proteins were refolded in presence of reducing agents and nucleophiles such as 2mM TCEP-HCl, 50 mM DTT (Roche-10708984001), and 0.5 M 250 Hydroxylamine (SRL-66164) with 1mM TCEP in renaturation buffer (55). For splicing and cleavage analysis, sample extraction at each time interval was followed by the addition of loading dye to stop the reactions and then boiling at $95^{\circ} \mathrm{C}$ for $5 \mathrm{~min}$. Resultant products from various refolding reactions were resolved through $4 \sim 10 \%$ gradient SDS PAGE. Protein bands were stained with Coomassie blue R-250 and densitometric analysis was performed by using GelQuant.Net biochemical solutions. Percentage(s) of splicing and cleavage products were 
measured by taking the percentage(s) of the ratio of the total splicing product (LE and I) over total proteins $(\mathrm{LE}+\mathrm{I}+\mathrm{P})$ and total $\mathrm{N}$-cleavage product $(\mathrm{NE}+\mathrm{NC})$ over total proteins $(\mathrm{NE}+\mathrm{NC}+\mathrm{P})$. The $0 \mathrm{hr}$ splicing value(s) were subtracted at each time point for baseline correction.

\subsection{Kinetic analyses}

Since Mtu 6X (His)-tagged WT SufB and SufB mutants (H-5A, H-38A, H-5A/H-38A, and 263 $\mathrm{C} 1 \mathrm{~A} / \mathrm{N} 359 \mathrm{~A})$ were purified and renatured at different temperatures, after normalizing splicing and cleavage values at different time intervals, the plot was generated by taking the percentage of splicing or cleavage product with respect to time (in min). Next, the curve was fitted in pseudofirst-order kinetics, with an equation $\mathrm{Y}=\mathrm{Y} 0+($ Plateau-Y0 $) *\left(1-\exp \left(-\mathrm{K}^{*} \mathrm{x}\right)\right)$ [Where $\mathrm{X}=$ time, $\mathrm{Y} 0$ $=\mathrm{Y}$ value when time $(\mathrm{X})=$ time 0 , Plateau $=\max \mathrm{Y}$ value at time $\mathrm{t}, \mathrm{K}=$ rate constant, expressed in reciprocal of the $\mathrm{X}$-axis (time units)] in graph pad prism software. The fitted curve was generated by automatic outlier elimination fitting in a nonlinear regression equation. The rate constant $(\mathrm{K})$ and Vmax were generated by the software. Half-life ( $\mathrm{t} 1 / 2)$ was calculated by graph pad prism using the formula $(\mathrm{Ln} 2 / \mathrm{K})$.

\subsection{Western blot}

Western blot analysis was performed using an anti-His antibody (Invitrogen, LOT 1902132) to confirm the identity of splicing and cleavage products. Following resolution through SDS PAGE, test proteins were transferred to a nitrocellulose membrane; at 50v, $2 \mathrm{~h}$. After a successful transfer, blocking was done with $5 \%$ skim milk for $2 \mathrm{~h}$ at room temperature. Then the blot was incubated with HRP conjugated anti-His antibody (Invitrogen, LOT 1902132) at 1:5000 dilutions for $16 \mathrm{~h}$ at $40 \mathrm{C}$. Then blot was washed with $1 \mathrm{X}$ TBST and developed using $13 \mathrm{ECL}$ as the substrate. N-extein detection was done with 1:2500 antibody dilution. 


\subsection{Mass spectrometry and chromatography}

After renaturation, proteins were resolved through 4 10\% SDS PAGE. Protein identification by mass spectrometry was performed at Central Proteomics Facility, Institute of Life Sciences, Bhubaneswar using the following standardized protocol. Well resolved protein gel bands were destained with $25 \mathrm{mM}$ ammonium bicarbonate $(\mathrm{ABC})$. Cleared gel bands were reduced with 20 mM DTT and then alkylated by $100 \mathrm{mM}$ iodoacetamide solution. The gel plugs were dehydrated sequentially with $50 \%, 100 \%$ acetonitrile $(\mathrm{ACN}$ ) and then digested with $0.3 \mu \mathrm{g}$ of trypsin (Absciex 4352157) in $25 \mathrm{mM} \mathrm{ABC}$ solution at $37^{\circ} \mathrm{C}$ for 16 hours. Peptides were extracted sequentially using extraction buffer containing $0.1 \%, 0.5 \%$, and $5 \%$ tri-fluoro acetic acid in $50 \% \mathrm{ACN}$ from gel bands. Extracted peptides were desalted by using C18 Zip-Tip (Millipore) and then dried in a centrifugal vacuum concentrator. Peptide samples were reconstituted with $0.1 \%$ TFA in $50 \%$ ACN solution mixed 1:1 ratio with $\alpha$-cyano-4-hydroxycinnamic acid solution $(\mathrm{C} 8982 ; 10 \mathrm{mg} / \mathrm{ml})$ and then spotted onto metal target plate in triplicates. MS and MS/MS spectra were acquired using MALDI TOF/TOF (AbSciex TOF/TOF 5800). Acquired spectra were searched against Mycobacterium tuberculosis from NCBInr database using mascot search engine in Protein Pilot Software for protein identification. Searches were performed allowing trypsin mis-cleavage up to Carbamido-methylation of cysteine, oxidation of methionine was also included as variable and fixed modification respectively. The peptide mass tolerance was 300 set as $100 \mathrm{ppm}$ for precursor ion and $0.8 \mathrm{Da}$ for fragment ion with +1 charge. Since all the splicing and cleavage products were identified as Mtu FL-SufB, the acquired spectra were researched against a customized database containing probable splicing products and cleavage products of SufB protein of Mycobacterium tuberculosis using mascot search engine in Protein Pilot Software with the same parameter. $\beta$-gal and BSA were run as internal calibration. The protein score, percent coverage, theoretical molecular weight, and Iso-electric $\mathrm{pH}$ value were obtained. The mass spectrometry proteomics 
data have been deposited to the ProteomeXchange Consortium via the PRIDE [1] partner repository with the dataset identifier PXD015199.

Purified and renatured Mtu FL-SufB protein was examined by analytical HPLC carried out on an Agilent 1200 series instrument equipped with a Zorbax gf-450 column $(6 \mu \mathrm{m}, 9.4 \times 250 \mathrm{~mm})$ with a flow rate of $1 \mathrm{ml} / \mathrm{min}$. All HPLC runs used the following solvent: 1:1 water and isopropanol (solvent 1 ) and $0.1 \%$ TFA (Trifluoroacetic acid) in water (solvent 2). The column was equilibrated using solvent 1 and solvent 2. A protein sample was inserted into the column and ran for 30 mins. The retention time vs protein intensity measured at $280 \mathrm{~nm}$ was noted. For reference, we ran $6 \mu 1$ of Precision plus protein ladder (Biorad1610374) diluted to 30 $\mu 1$ using Sodium phosphate buffer. The retention time vs molecular weight of the known protein standards was measured and plotted to make a standard curve. The unknown protein peaks from the test samples were compared with the standard curve to find out the relative molecular weights. The molecular weights of the expected fragments from the MALDI-TOF/TOF MS (Table S3) data were compared with the standard curve and molecular weights of the unknown peaks were determined.

Molecular weight of the SufB DM (H5A/H38A) was determined by FPLC. H215A/H248A SufB mutant protein $(\mathrm{DM})$ was isolated and purified under denaturing conditions. The relative molecular weight of DM was confirmed by Gel filtration chromatography using Hi prep 16/60 Sephacryl S200 GPC column (HiPrep 16/60 GE Healthcare, Cat. no. 10255928). 8M Urea buffer was removed from the protein sample by dialysis in $20 \mathrm{mM}$ sodium phosphate and $0.15 \mathrm{M}$ sodium chloride buffer with $\mathrm{pH}$ 7.4. Then the sample was concentrated using Protein concentrator (Thermo Scientific 88515). For FPLC analysis, the column was equilibrated with 2 column volume (cv) of sodium phosphate buffer ( $\mathrm{pH}$ 7.4) followed by loading of molecular weight standard Bovine Serum Albumin (BSA) at a concentration of $1 \mathrm{mg} / \mathrm{ml}$. Then $2 \mathrm{mg}$ of purified DM protein was loaded 
onto the GPC column. The run was performed at a flow rate of $1 \mathrm{ml} / \mathrm{min}$ while keeping the temperature at $20^{\circ} \mathrm{C}$. The fractions eluted at an absorbance of $280 \mathrm{~nm}$ were collected and analyzed by SDS-PAGE under reducing and non-reducing conditions.

\subsection{Molecular Dynamics (MD) Simulations}

\subsubsection{Homology modeling of Mtu SufB precursor}

A homology model of the Mtu FL-SufB precursor was built using the SufB chain of the E. coli SufB-SufC-SufD complex (PDB ID: 5AWF, chain A), the $S$. cerevisiae intein homing endonuclease (PDB ID: 1VDE, chain A), and the T. kodakarensis homing endonuclease (PDB ID: 2CW7, chain A) using Bioluminate 2.7 from Schrödinger. The model was assessed by Ramachandran plot analysis and PROSA(Z-score) (56).

\subsubsection{MD simulation analysis}

Explicit-solvent MD simulations were performed using GROMACS (V5.1.4) (57) with the OPLSAA force field (58) and the SPC/E water model $(59,60)$. The Mtu SufB model (846 aa) was solvated in a cubic box with at least $1 \mathrm{~nm}$ distance between the protein and the edge of the box, and neutralized with $\mathrm{NaCl}$. The system was minimized with target Fmax of no greater than 1000 kJ mol-1 $349 \mathrm{~nm}-1$ with steepest descent minimization with a spherical cut-off at $1 \mathrm{~nm}$ was imposed on all intermolecular interactions with verlet cut-off scheme $(61,62)$. The leap-frog algorithm with a timestep of $2 \mathrm{fs}$ (63) and a canonical NVT ensemble was used to run the simulation for $100 \mathrm{~ns}$, with temperature maintained at $300 \mathrm{~K}$ through velocity-rescale coupling and no temperature coupling. H-bonds were constrained using lincs with the order of 4 (64). The particlemesh Ewald (PME) algorithm was used for implementing long-range electrostatic interactions with the grid dimension of $0.16 \mathrm{~nm}$ and interpolation order of 4 (65). Histidine at positions 5 and 
36 were mutated to alanine by homology modelling, and MD simulations were performed on these mutant proteins in an identical fashion. The MD simulation trajectories were analyzed with GROMACS and Pymol, and plotted using Origin 8.0.

\section{RESULTS}

\subsection{Structural domains of Mtu full-length (FL) SufB precursor}

We obtained the protein sequences for Fe-S cluster assembly protein SufB [Mycobacterium tuberculosis H37Rv], an 846 amino acid protein (Accession number WP_003407484.1, GI: 397673309) and the intein-less SufB protein [Mycobacterium smegmatis MC2 155], a 477 amino acid protein (Accession number YP_887437.1, GI: 118472504) from NCBI protein database. A pair-wise sequence alignment using the ClustalW server very clearly delineated the intein sequence as well as identified $\sim 95 \%$ sequence similarities between the extein sequences. A blast search against the sequences deposited in Inbase also identified the intein sequence as 359 amino acid Mtu SufB (Mtu Pps1) intein From M. tuberculosis strains H37Rv. Based on sequence alignment and structural analyses of SufB intein and homing endonuclease domain I-CreI and intein homing endonuclease Ii (PDB: 2CW7), the final demarcation of different structural domains of M. tuberculosis and their respective sequences appeared to be as shown in Table S2.

Residues encompassing N- \& C-terminal extein and intein sequences from Mtu SufB precursor are illustrated in Figure 1A. Multiple sequence alignment of Mtu SufB precursor and other intein bearing proteins highlighted conservation of catalytic cysteines $(\mathrm{C} 1$ and $\mathrm{C}+1)$, Block B His67, Block F Asp77, penultimate His358, and terminal Asn359 in the intein and extein regions (Figure 1B). Hence, SufB precursor protein (846 aa) is likely to follow a canonical intein splicing pathway to generate the following products (Figure 1C); ligated N-(252aa) and C-(235 aa) exteins 


\section{B}

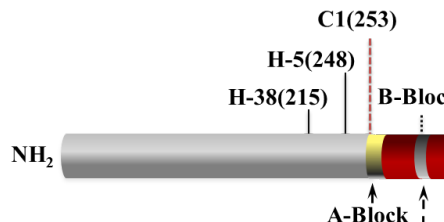

[TXXH] [CC(611aa) and C-extein (CE)] $(51,66)$.

\section{A}

A

to form native SufB protein (487aa), SufB intein (359aa) with splicing domain (N- and C-terminal inteins; 155aa) and endonuclease domain (204aa). Off-pathway products may give rise to Nterminal cleavage products $[\mathrm{NC}(594 \mathrm{aa})$ and $\mathrm{N}$-extein $(\mathrm{NE})]$ and C-terminal cleavage products 
C

(i)

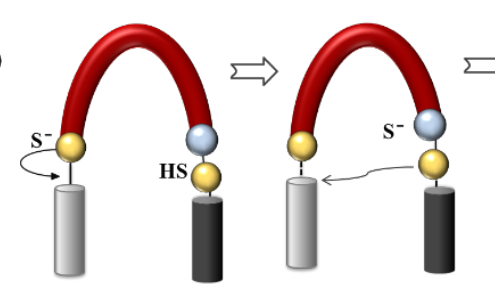

(ii)

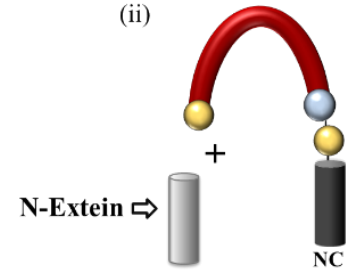

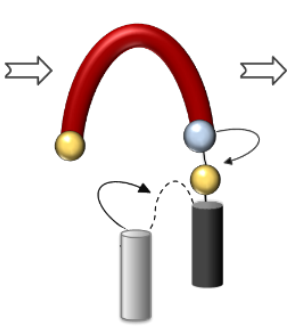

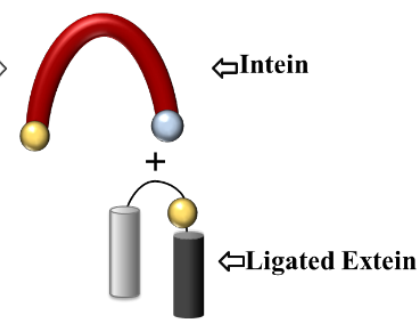

(iii)

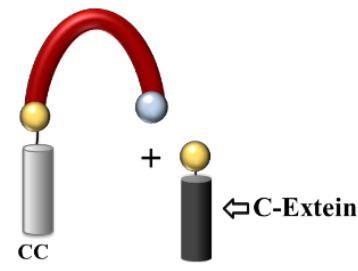

Cysteine OAsparagine - Peptide bond -- Thioester bond $\mathrm{NC}=\mathrm{N}$-terminal Cleavage $\mathrm{CC}=\mathrm{C}$-terminal Cleavage

Figure 1. (A) Schematic representation of different structural domains of Mtu full-length SufB precursor. The conserved and the catalytic residues in the FL-SufB protein sequence are shown at the top. Further, numbering for different structural domains are also displayed; intein residues $(1,2 \ldots \ldots, n)$, N-extein residues $(-1,-2, \ldots \ldots-n)$ and $\mathrm{C}$-extein residues $(+1,+2, \ldots \ldots+\mathrm{n})$. Catalytic cysteines responsible for sequential nucleophilic displacement reactions are $\mathrm{C} 1$ and $\mathrm{C}+1$, terminal intein residue responsible for branched intermediate resolution is $\mathrm{N} 359$, conserved histidines in the N-extein region are H-5 (248, full-length protein) and H-38 (215, full-length protein). G+234 is the last residues of Mtu SufB, (B) Multiple sequence alignment of Mtu SufB with other intein bearing species shows conservation of critical residues (highlighted in different colors) that participate in a canonical splicing pathway. (C) Schematic diagram showing the mechanism of intein splicing. (i) There are four major reaction steps; first two steps of sequential nucleophilic attack by $\mathrm{C} 1$ and $\mathrm{C}+1$ generate branched intermediate by trans-esterification. Next, cyclization of terminal Asn resolves the branched intermediate, ligates exteins and cleaves off SufB intein, (ii) and (iii) represent off-pathway products from protein splicing; $\mathrm{N}$ - and C-terminal cleavage products respectively.

\subsection{Conservation of His-5 and His-38 in different bacterial species}

Our sequence analysis results indicate that SufB from different mycobacterial and archeal species exhibits a high degree of sequence similarities (Figure 2A and B). This is further supported by phylogenetic analysis to show that these mycobacterial proteins possibly have a common ancestral origin (67). Analysis cladogram of SufB intein sequences from different mycobacterial species shows significant similarities, except in M. leprae, M. lepromatosis, M. triplex and $M$. 
bioRxiv preprint doi: https://doi.org/10.1101/2021.07.07.451452; this version posted August 16, 2021. The copyright holder for this preprint (which was not certified by peer review) is the author/funder. All rights reserved. No reuse allowed without permission.

Xenopi, possibly due to different intein insertion sites (39). Divergence is also noticed in Ferroplasma for intein [Figure 2C (ii)] and precursor sequence [Figure 2C (i)] perhaps due to differences in the kingdoms. The discrepancies in the phylogenetic tree analyses of FL-SufB precursor proteins suggest that there are variations in the extein sequences and intein insertion sites. This suggests intein evolution and independent intein transfer in different species and kingdoms.

A

\begin{tabular}{|c|c|c|c|c|c|c|c|}
\hline M.tuberculosis & $06)$ & ------ & & $---S F I Y V P$ & PGVHVDIPLQ & AYEL & \\
\hline icroti & 6) & --------- & ----- & ----SFIYVP & PGVHVDIPLQ & AYFRINTENM & \\
\hline fricanum & 5) & --------- & ---------- & $----S F I Y V P$ & PGVHVDIPLQ & YFRINTENM & \\
\hline pvis & 5) & --------- & ---------- & ----SF IYVP & DIPLQ & NM & \\
\hline rgelatii & & --------- & ---------- & $----S F I Y V P$ & IPLQ & NM & \\
\hline & & ---------- & ---------- & $----S F I Y V P$ & LQ & NM & \\
\hline & & ---------- & ---------- & $----S F I Y V P$ & DIPLQ & AYFF & \\
\hline i W & & ---------- & --------- & ----SFIYVP & DIPLQ & $\mathrm{AYEl}$ & \\
\hline p & & ---------- & ---------- & $----S F I Y V P$ & DIPLQ & $\mathrm{AYFF}$ & $(22$ \\
\hline$=i i$ & 1 & ---------- & ---------- & $----S F I Y V P$ & LQ & $\mathrm{AYFl}$ & 18 \\
\hline M. & 206 & -------- & --- & $----S F I Y V P$ & DIPLQ & AYF & $(2$ \\
\hline peddii & $(20$ & ---------- & ---------- & $----S F I Y V P$ & DIPLQ & $\mathrm{AYF}$ & (2. \\
\hline M.s & $(57$ & ----------- & ---------- & $----S F I Y V P$ & JEIPLQ & AYFR & \\
\hline Lex & ' & ---------- & ---------- & $----S F I Y V P$ & VDIPLQ & AYFR & \\
\hline M. 1 & ( & :KPTYDIQVV & GLENFVANGI & VAHNSFIYVP & VDIPLQ & AYFR & \\
\hline natosis & $(56$ & EKPTYDIQVV & GLENFVANGI & VAHNSF IYVP & PGV & AYFR & \\
\hline Ferroplasma & (196) & ---------- & ---------- & ----SFLYVP & KNVT I DMPLQ & TYFRMNGEQS & \\
\hline
\end{tabular}

M.leprae (614)
M.tuberculosis (232)

$\begin{array}{ll}\text { M.microti } & \text { (232) } \\ \text { M.africanum } & \text { (232) }\end{array}$

M.bovis (232)

M.Bourgelatii (225)

M.Kansasii (225)

M.orygis (232)

M.gastri_W (225)

$\begin{array}{ll}\text { M.gastrip } & (479) \\ \text { M. canettii } & \text { (237) }\end{array}$

$\begin{array}{ll}\text { M.canettii } & \text { (237) } \\ \text { M.caprae } & \text { (232) }\end{array}$

M.Pinnipeddi (232)

M.xenopi (604)

M.triplex (603)

M.lepromatosis (613)

Ferroplasma

\begin{tabular}{|c|c|c|c|c|c|}
\hline $2+\operatorname{LNL}$ & EGSYVHYVE & LIT & PIE & SIRVGDFVTG & \\
\hline GQFERTLIIA & DEGSYVHYVE & LIT & TADGDLRPIE & SIRVGDFVTG & \\
\hline TLIIA & DEGS & LIT & PIE & VTG & \\
\hline LIIA & YVE & & PIE & TG & \\
\hline LIIA & $\mathrm{DEC}$ & & IE & TG & \\
\hline LIIA & $\mathrm{DEC}$ & & IE & SS & \\
\hline LIIA & $\mathrm{DEC}$ & & & & \\
\hline LIIA & $\mathrm{DEC}$ & & & & \\
\hline LIIV & GEN & & --- & ---- & \\
\hline LIIA & $\mathrm{DEC}$ & IT & PIE & SI & \\
\hline LIIA & $\mathrm{DEC}$ & {$[\mathrm{T}$} & $\mathrm{TA}$ & SI & \\
\hline RTLIIA & DEGS & AGELIT & TADGDLRPIE & SIRVGDFVTG & \\
\hline GQ & $\mathrm{DEl}$ & ---ー-- & ---------- & --------- & \\
\hline LIIV & $\mathrm{DE}$ & -- & ---------- & ---------- & \\
\hline LIIA & DTG & ----0 & ---------- & ---------- & \\
\hline & & -------- & ---------- & ---------- & \\
\hline 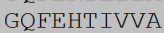 & & LPEDELIS & QGDkfv-PIN & ELLTDDSVVS & \\
\hline
\end{tabular}

B
M.tuberculosis

M.marinum

M. fortuitum

M. smegmatis

M.shigaense

S. aureus

B.subtilis

M. tuberculosis

M.marinum

M. fortuitum

M. smegmatis

M. shigaense

S. aureus

B.subtilis
(181) EYFGTVIPAGDNKFSALNTAVWSGGSF IYVPPGVHVDIPLQAYFRINTENMGQFERTLII (240) (174) QYFGTVIPAGDNKFSALNTAVWSGGSF IYVPPGVHVDIPLQAYFRINTENMGQFERTLII (245) (181) KYFGTVIPAGDNKFSALNTAVWSGGSFIYVPPGVHVDIPLQAYFRINTENMGQFERTLII (251) (171) QYFGTVIPAGDNKFSALNTAVWSGGSFIYVPPGVHVDI PLQAYFRINTENMGQFERTLI I (241) (175) QYFGSVIPAGDNKFSALNTAVWSGGSF IYVPPGVHVDIPLQAYFRINTENMGQFERTLII (245) (159) KYFASVVPAADNKFAALNSAVWSGGSFIYVPKNIKLDTPLQAYFRINSENMGQFERTLII (229) (159) EHWAKVIPPTDNKFAALNSAVWSGGSF IYVPKGVKVETPLQAYFRINSENMGQFERTLII (229) 
C

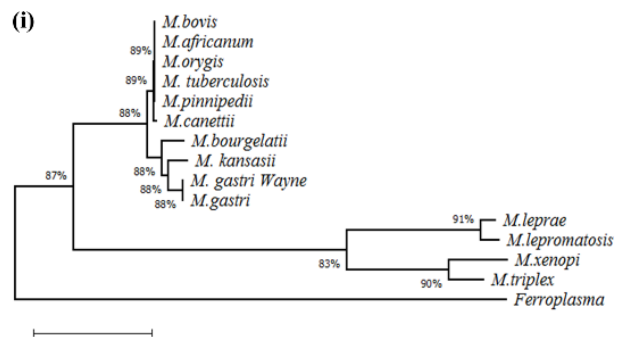

0.21

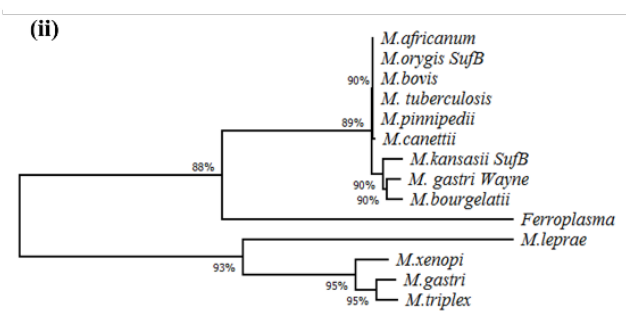

0.2

Figure 2. Histidine conservation in the $\mathbf{N}$-extein region of Mtu SufB protein (A) Multiple sequence alignment of $\mathrm{SufB}$, focusing on $\mathrm{N}$-extein intein junction in mycobacterial and archeal species. Conserved histidine residues at -5 and -38 positions in the $\mathrm{N}$-extein region are highlighted in yellow and green respectively. Catalytic $\mathrm{C} 1$ of inteins are marked in red. N-extein and intein residues are delineated by shading in grey and cyan blue, (B) Multiple sequence alignment of SufB, focusing on N-extein intein junction in $M t u$ and other intein-less mycobacteria and in organisms where SUF is the exclusive system for Fe-S cluster assembly, (C) (i) \& (ii) phylogenetic analyses of full-length (FL) SufB protein and SufB intein sequences in mycobacteria and Ferroplasma acidarmans respectively.

Further, two conserved histidines were identified in the N-extein region of different mycobacterial and archaeal SufB precursor proteins, His-5 and His-38 (Figure 2A). His-5 is also conserved in intein-less SufB proteins from Staphylococcus aureus, Bacillus subtilis, and certain mycobacteria that use SUF complex as the sole pathway for [Fe-S] cluster generation (Figure 2B) $(68,69)$. Although His-38 is conserved in both intein-bearing and intein-less mycobacterial SufB proteins, conservation is missing in intein-less bacterial systems like Staphylococcus aureus and Bacillus subtilis where SUF system is essential to synthesize [Fe-S] clusters (Figure 2B).

Although His-5 is proximal to the N-terminal splice junction in the protein sequence our MD simulations results (explained later in this manuscript) detected the proximity of $\mathrm{H}-38$ to catalytic Cys1 in the predicted structural model for Mtu SufB. Identification of two highly conserved metal-chelating residues proximal to $\mathrm{N}$-terminal extein intein junction raises the possibility of 
their regulatory roles on cleavage and/or splicing as well as in the functionality of [Fe-S] cluster assembly protein SufB. Despite their remote positions these two histidines possibly come closer to the active site and participate in protein splicing either via direct or indirect interaction with the catalytic residues near cleavage site(s). A comprehensive kinetic study of in-vitro splicing, and $\mathrm{N}$-cleavage reactions are presented in the next section. Further mechanistic understanding of the roles of highly conserved histidines was explored via molecular dynamics (MD) simulations as shown later in this manuscript.

\subsection{Kinetic study to evaluate the roles of conserved His-5 and His-38 on Mtu SufB intein}

\section{splicing}

Plasmid constructs for the current work were engineered as mentioned in the materials and methods section. Detection of two highly conserved His-5 and His-38 in the N-extein region raises the possibility of their regulatory effect on $M t u$ SufB intein cleavage and/or splicing either by interaction with catalytic residues directly or indirectly via other active site residues. To test this hypothesis, alanine substitution was made in place of His-5 and His-38 to generate single mutants (H-5A, H-38A) and double mutant (H-5A/H-38A, DM) (Materials and Methods). Alanine substitutions for active site residues have resulted in a complete blockage of cleavage and splicing reactions as shown by earlier studies (30). The negative control used for the above study was splicing inactive (SI) SufB double mutant. Substitution of both C1 and N359 to alanine (C1A/N359A) is expected to abolish intein splicing completely (70).

The above proteins were purified under denaturing conditions and refolded at $20^{\circ} \mathrm{C}$ as described in the materials and methods. FL-SufB precursor, H-5A and H-38A SufB mutants gave rise to the precursor $(\mathrm{P}, 95.98 \mathrm{KDa}), \mathrm{N}$-terminal cleavage $(\mathrm{NC}, 66 \mathrm{KDa})$ product, N-extein $(\mathrm{NE}$, 
29.9KDa), ligated exteins (LE, 55.7 KDa), and intein (I, 40.2 KDa) upon in vitro refolding (Figure 3). As predicted none of these products were seen in the case of SI double mutant [Figure S2E (i)] and transformants expressing empty vector pACYC Duet-1 [Figure S2E(ii)]. C-terminal cleavage product $(\mathrm{CC}, 70.12 \mathrm{KDa})$ and $\mathrm{C}$-extein $(\mathrm{CE}, 25.76 \mathrm{KDa})$ were not detected by SDS PAGE possibly due to protein degradation. Consequently, we were unable to analyze the effects of His-5Ala and His-38Ala mutation on the C-terminal cleavage reaction of Mtu SufB. However, we did confirm the presence of P, LE, NE, and CC products via western blot (Figure 3B).

478

A

(ii)

(i)

(iii)

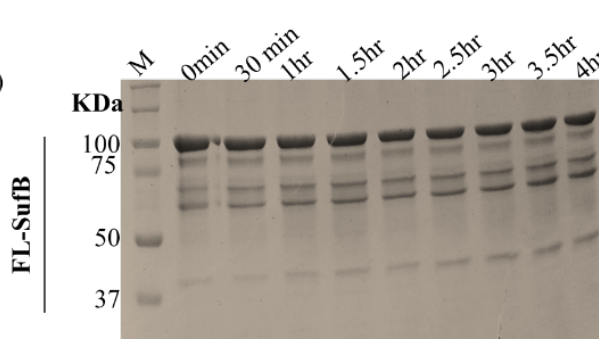

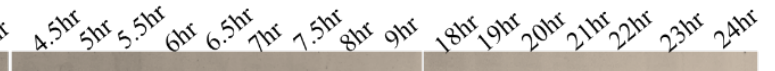

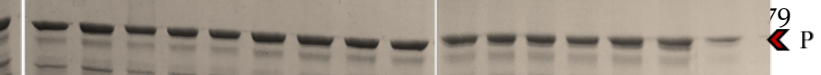
ニニニニニニニニニーニーニニニー $\mathrm{R} \mathrm{NC}$
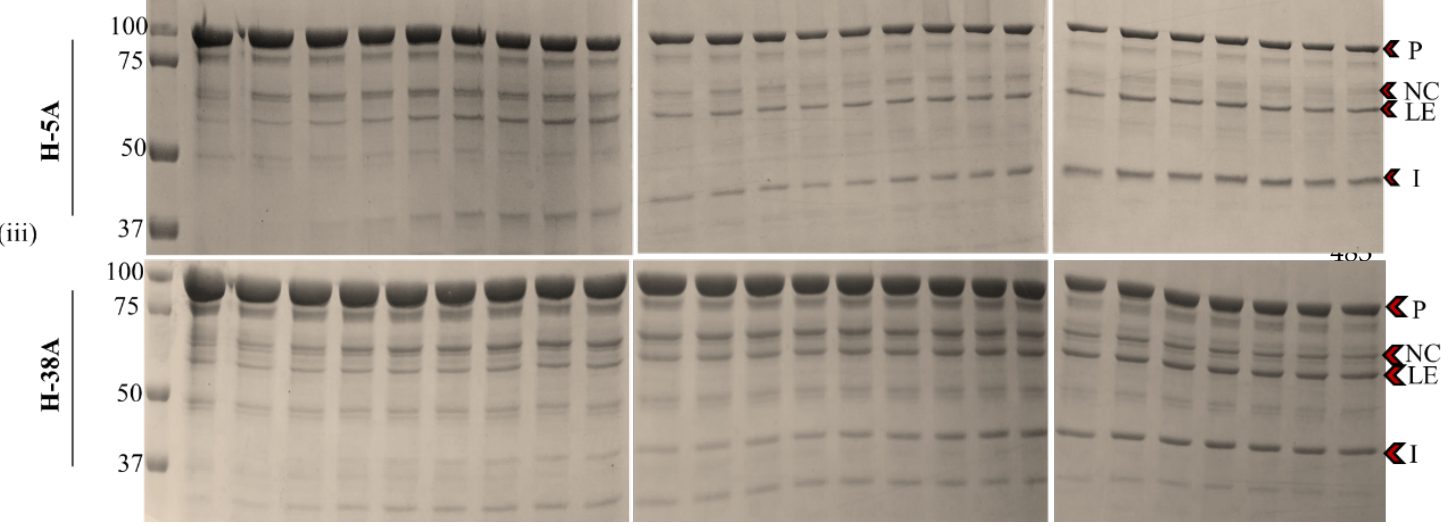
B (i)

(ii)

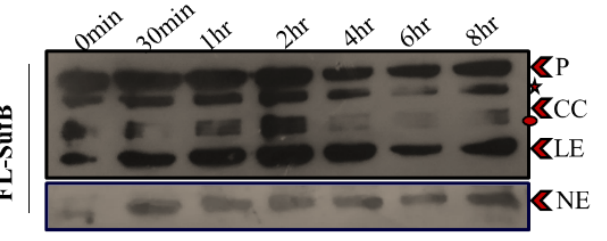

(iii)

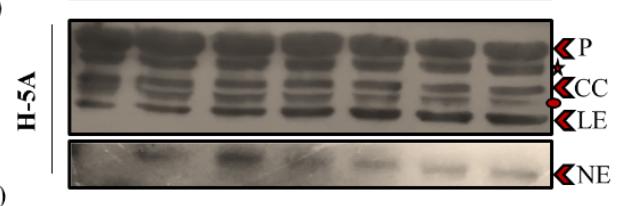

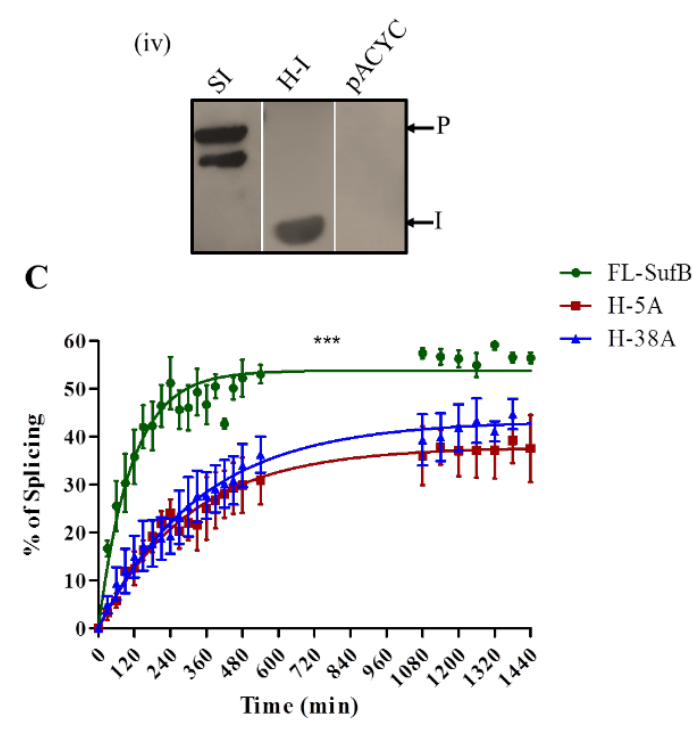

$\star$ Possible precursor degradation product

- Off-pathway splicing intermediate
Figure 3. Effect of H-5 and H-38 mutation on Mtu SufB splicing. (A) Products from in-vitro refolding reactions were resolved through $4 \sim 10 \%$ gradient SDS PAGE as shown in, (i) FL-SufB precursor, (ii) H-5A and (iii) H-38A SufB mutant proteins exhibiting splicing over different time periods, (B) Western blot to confirm the identity of splicing and cleavage products for (i) FLSufB precursor (ii) H-5A iii) H-38A SufB mutants and iv) controls; SI (splicing inactive double mutant SufB), H-I[6x(His)- tagged SufB intein], pACYC Duet-1(cell lysates expressing empty expression vector). Anti-His antibodies detected presence of $6 \mathrm{x}$ (His)- tagged $\mathrm{P}, \mathrm{CC}, \mathrm{LE}$ and NE. $\mathrm{NE}$ is blotted separately with higher concentration of primary antibody. (C) Kinetic study of protein splicing in $M t u$ FL-SufB precursor, H-5A and H-38A SufB mutant proteins. Splicing products were quantified and plotted over different time periods and the curve was fitted to a pseudo first order reaction; $\mathrm{Y}=\mathrm{Y} 0+\left(\right.$ Plateau-Y0) $*\left(1-\exp ^{\left(-\mathrm{K}^{*} \mathrm{x}\right)}\right)$. All the experiments were performed in triplicates and error bars represents $( \pm 1)$ SEM from 3 independent sets of experiments. A comparative analysis demonstrates statistically significant difference in splicing efficiency $(\mathrm{p}<0.0001)$ in FL-SufB precursor, H-5A and H-38A mutant SufB. P=Precursor, $\mathrm{CC}=\mathrm{C}$-cleavage, $\mathrm{NC}=\mathrm{N}$-cleavage, $\mathrm{LE}=$ Ligated Extein, $\mathrm{I}=$ Intein, $\mathrm{NE}=\mathrm{N}-$ Extein, $\mathrm{M}=$ Protein marker. 


\section{$\underline{\text { 3.3.1 His-5Ala and His-38Ala SufB mutants exhibit attenuated splicing reaction }}$}

513 Splicing efficiency was determined following in vitro renaturation of FL- and mutant SufB

514

515

516

517

518

519

520

521

522

523

524

525

526

527

528

529

530

531 proteins $(\mathrm{H}-5 \mathrm{~A}$ and $\mathrm{H}-38 \mathrm{~A})$ over a period for $24 \mathrm{~h}$. Precursor $(\mathrm{P})$ proteins detected at time $0 \mathrm{~h}$ were quite comparable for FL- and mutant proteins (Figure 3A). Ligated extein fractions were visualized at $0 \mathrm{~h}$ as a possible soluble contaminant and accumulated product of protein purification. Densitometric analysis of protein products was performed via Gelquant.NET biochemical solutions. Splicing efficiency was calculated as a percentage of splicing $[(\mathrm{I}+\mathrm{LE} / \mathrm{P}+\mathrm{I}+\mathrm{LE}) \times 100]$.

Splicing efficiency was plotted over time as pseudo-first-order reaction kinetics in GraphPad Prism version 9.0 or 8.0 (Figure 3C). At $20^{\circ} \mathrm{C}$, WT FL-SufB shows a splicing efficiency ( $\left.\mathrm{Y}_{\max }\right)$ of $(53.7 \pm 1.31) \%$ with a rate constant $(\mathrm{K})$ value of $\left(9.92 \pm 0.001 \times 10^{-3} \mathrm{~min}^{-1}\right)$ and a half-life $\left(\mathrm{t}_{1 / 2}\right)$ of 1.1h (Table 1). Under the same experimental conditions, H-5A SufB mutant splices out with maximum efficiency $\left(\mathrm{Y}_{\max }\right)$ of $(37.8 \pm 0.68 \%)$, a rate constant $(\mathrm{K})$ value of $\left(3.3 \pm 0.0001 \times 10^{-3} \mathrm{~min}^{-}\right.$ $\left.{ }^{1}\right)$ and a half-life $\left(t_{1 / 2}\right)$ of 3.4 h. H-38A SufB mutant splices out with an efficiency $\left(Y_{\max }\right)$ of (43.35 $\pm 1.7 \%)$, the rate constant $(\mathrm{K})\left(2.9 \pm 0.0003 \times 10^{-3}\right)$, and half-life $\left(\mathrm{t}_{1 / 2}\right) 3.8 \mathrm{~h}$. This clearly demonstrates a 3 fold and 3.4-fold reduction ( $\mathrm{p}<0.0001$; one-way ANOVA) in splicing efficiency for $\mathrm{H}-5 \mathrm{~A}$ and $\mathrm{H}-38 \mathrm{~A}$ mutants, respectively, relative to FL-SufB precursor. These observations further strengthen our hypothesis that histidines at -5 and -38 positions may have regulatory role on Mtu SufB intein splicing. 
Table 1. Comparative analysis of different kinetic parameters for splicing in FL-SufB precursor, $\mathrm{H}-5 \mathrm{~A}$ and $\mathrm{H}-38 \mathrm{~A}$ SufB mutant proteins, at $20^{\circ} \mathrm{C}$ temperature. These data were extracted from Figure 3C.

\begin{tabular}{|c|c|c|c|}
\hline Kinetic parameters & FL-SufB & H-5A & H-38A 537 \\
\hline $\mathbf{Y}_{\max }$ & $53.81 \pm 1.03$ & $37.82 \pm 1.68$ & $43.35 \pm 1.7 \quad 538$ \\
\hline$\underset{\left(\text { min }^{-1}\right)}{\text { Rate constant }}(\mathbf{K})$ & $9.92 \pm 0.007 \times 10^{-3}$ & $3.3 \pm 0.0003 \times 10^{-3}$ & $2.9 \pm 0.0003 \times 10_{539}^{-3}$ \\
\hline Half-life $\left(t_{1 / 2}\right)$ & $1.1 \mathrm{~h}$ & $3.4 \mathrm{~h}$ & $3.8 \mathrm{~h}$ \\
\hline
\end{tabular}

Further analysis was done by the kinetic study of H-5A/H-38A SufB DM under similar experimental conditions. Surprisingly, H-5A/H-38A DM expression gave rise to a faint precursor (P) band and a truncated product of 28KDa size detected by SDS PAGE analysis whereas western blot detected both the $\mathrm{P}$ and the truncated protein (Figure 4 and Figure S2). The 28KDa product was confirmed by FPLC as well (Figure S3). The possibility of a frameshift during cloning was ruled out by repeat sequencing. A comparative analysis of splicing and cleavage products for FLSufB, N359A SufB (cleavage mutant with isolated NC products), C+1A SufB (cleavage mutant with isolated NC products), and H-5A/H-38A SufB DM provided further clarification (Figure 4). The 28KDa $(\mathrm{H}-5 \mathrm{~A} / \mathrm{H}-38 \mathrm{~A}$ SufB DM) truncated protein was identified as a possible splicing intermediate or a product of protein degradation, but different from the N-extein (29.9KDa). 
$\mathbf{A}$

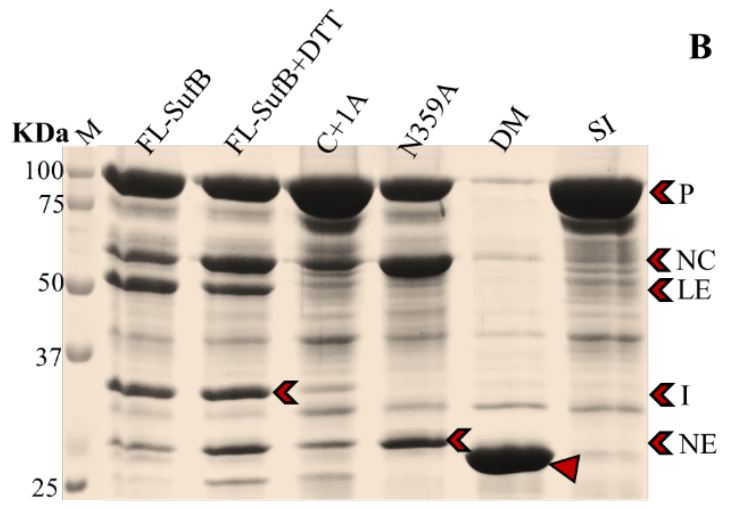

B

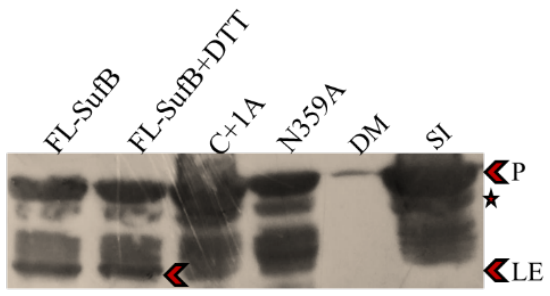

$\star$ Possible precursor degradation product

$\triangle 28 \mathrm{KDa}$ truncated protein product for H-5A/H-38A SufB Double mutant(DM)

Figure 4. Identification of Mtu H-5A/H-38A SufB double mutant(DM): (A) SDS Gel showing splicing and N-cleavage reactions in Mtu FL-SufB precursor, C+1A, N359A, DM (H5A/H-38A) and SI (C1A/N359A) SufB mutant (B) Confirmation of protein products for FLSufB, C+1A, N359A, DM (H-5A/H-38A) and SI (C1A/N359A) SufB mutant proteins via immuno- blotting assay. Anti-His antibodies detected presence of $6 \mathrm{X}(\mathrm{His})$ tagged $\mathrm{P}$ and LE. NE and SufB DM truncated protein product are blotted separately with higher concentration of primary antibody. $\mathrm{P}=$ Precursor, $\mathrm{NC}=\mathrm{N}$-cleavage, $\mathrm{LE}=$ Ligated Extein, $\mathrm{I}=\mathrm{Intein}, \mathrm{NE}=\mathrm{N}$-Extein.

\subsection{Kinetic study to assess the roles of His-5 and H-38 in the first amide and thioester} equilibrium

Next, we examined TCEP [Tris(2-carboxyethyl) phosphine], DTT (Dithiothreitol), and HA (Hydroxylamine) induced N-cleavage reactions in FL- and mutant SufB proteins. DTT can act both as a nucleophile and reducing agent. Hydroxylamine acts as a nucleophile and like DTT was used as a control for DTT and HA-induced N-cleavage reactions (72). 
Since the optimum temperature for FL- SufB splicing is found to be $20^{\circ} \mathrm{C}$, N-cleavage reactions for the test proteins were also conducted at the same temperature. Purified proteins were renatured in presence of different reducing agents, DTT $(50 \mathrm{mM}), \mathrm{HA}(0.5 \mathrm{M})$, and TCEP( $2 \mathrm{mM})$ for different durations of time (materials and methods). Products of N-cleavage reactions were visualized by $4 \% \sim 10 \%$ gradient SDS PAGE (Figure 5, 6, and Figure S4) and densitometric analysis of the cleavage products was performed by Gelquant.NET biochemical solutions. The percentage of $\mathrm{N}$-cleavage reaction product was calculated as $[(\mathrm{NE}+\mathrm{NC} / \mathrm{P}+\mathrm{NE}+\mathrm{NC}) \times 100]$.

\subsubsection{H-5A and H-38A SufB mutants displav reduced TCEP mediated $N$-cleavage reaction}

TCEP mediated reaction was analyzed for $4 \mathrm{~h}$ in FL- SufB and mutant SufB proteins. The mutant proteins clearly exhibited diminished production of N-cleavage (NC) products relative to FLSufB [Figure 5, Table 2(i)]. Reaction mediated by TCEP did not give a proper curve that could be fitted in linear or non-linear regression. In FL- SufB, \% N-cleavage was accelerated until 40 min followed by a decline as splicing became distinct. H-5A and H-38A SufB mutants presented a sluggish course with the $\mathrm{N}$-cleavage product peak at 60-80 min and then tapered off. At $40 \mathrm{~min}$ and $60 \mathrm{~min}, \mathrm{H}-5 \mathrm{~A}$ displayed 2 -fold and 1.5 -fold diminution in $\% \mathrm{~N}$-cleavage respectively, relative to FL-SufB. Likewise, at $40 \mathrm{~min}$ and $60 \mathrm{~min}, \mathrm{H}-38 \mathrm{~A}$ mutant displayed a 3.8 -fold and 3-fold reduction in $\%$ of N-cleavage respectively relative to FL-SufB (Figure 5). 
A

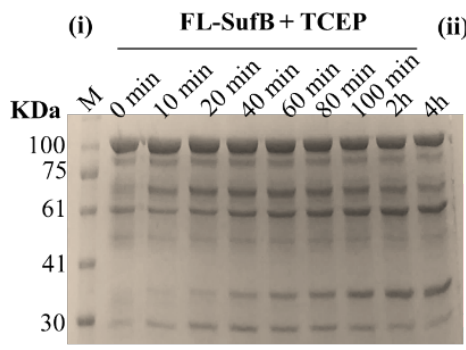

(ii)

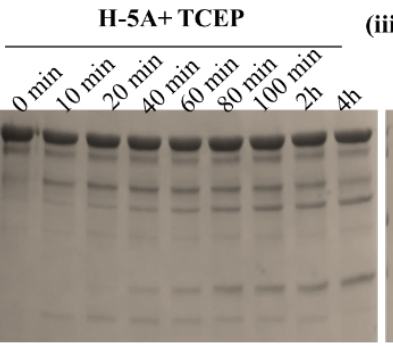

(iii)

H-38A+ TCEP

B

Figure 5. Effect of H-5A and H-38A mutation on TCEP mediated N-cleavage reaction in Mtu SufB: (A) SDS Gel image of (i) FL-SufB precursor (ii) H-5A and (iii) H-38A SufB mutant proteins displaying $\mathrm{N}$-cleavage reactions over different time periods; $\mathrm{B}$ ) (i) Comparative analysis of TCEP mediated reaction in FL-SufB, H-5A and H-38A SufB mutant proteins, indicates statistically significant differences $(\mathrm{P}=0.0011$, one way ANOVA) in $\%$ of $\mathrm{N}$ cleavage over different time periods. Reaction mediated by TCEP did not give a proper curve which could be fitted in linear or non-linear regression. All the experiments were performed in triplicates and error bars represent $( \pm 1) \mathrm{SEM}$. $\mathrm{P}=$ Precursor, $\mathrm{NC}=\mathrm{N}$-cleavage, LE=Ligated Extein, I=Intein, $\mathrm{NE}=\mathrm{N}$-Extein, $\mathrm{CC}=\mathrm{C}$-Cleavage. performed. The extracted data for HA-induced reactions were plotted over different time frames 
and the curve was fitted to a pseudo-first-order reaction with the equation, $\mathrm{Y}=\mathrm{Y} 0+(\mathrm{Plateau}-\mathrm{Y} 0)$ *(1-exp $\left.{ }^{\left(-K^{*}\right)}\right)$, (Figure 6). H-5A and H-38A mutants exhibited about 1.4-fold and 1.5-fold reduction ( $\mathrm{p}=0.0025$; one-way ANOVA) in $\%$ of N-cleavage, respectively, relative to FL- SufB although the $\mathrm{K}$ and $\mathrm{t}_{1 / 2}$ values were comparable [Figure 6C, Table 2 (ii)]. HA is an alpha nucleophile that intercepts between amide-ester equilibrium and induces $\mathrm{N}$-cleavage. It enhances the nucleophilicity of residues that leads to N-cleavage $(73,74)$. The mutation effect on HAinduced N-cleavage reaction was similar to what is observed in presence of TCEP. However, no significant differences in N-cleavage efficiency were noticed between H-5A, H-38A mutants, and FL- SufB in presence of DTT (Figure S4 and Table S4). Similar to HA, DTT is also a nucleophile that enhances the nucleophilicity of residues and facilitates $\mathrm{N}$ - terminal cleavage reaction $(72,75)$. Besides, DTT interacts directly with the residues that may increase the flexibility of the active site structure (76). Possibly, this abolishes the effect of H-5A and H-38A mutation on N-terminal cleavage. Supported by our current results we hypothesize that histidines at -5 and -38 positions in the $\mathrm{N}$-extein region may have a role in Cys 1 activation directly or via structural alignment of other active site residues. This likely explains why catalytic cysteines Cys 1 and $\mathrm{Cys}+1$ are interacting differently in His mutants, hindering the $\mathrm{N}$-cleavage reaction. Affirmation of the above hypothesis was done by MD simulations (upcoming section).

.


A

651

653

654

655

656

657

658

659

660

661

662

663

664

665

666

667

668

669

670

671

672

673 (i)

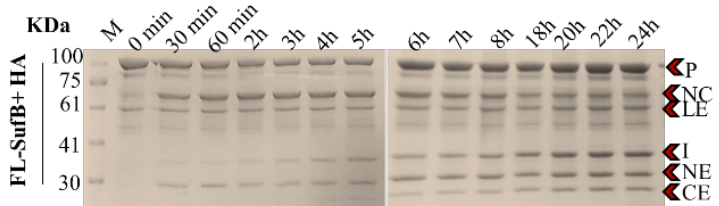

B

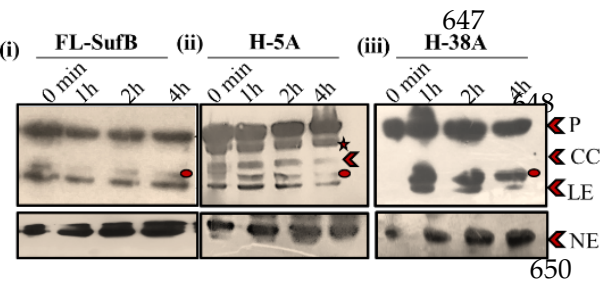

(ii)
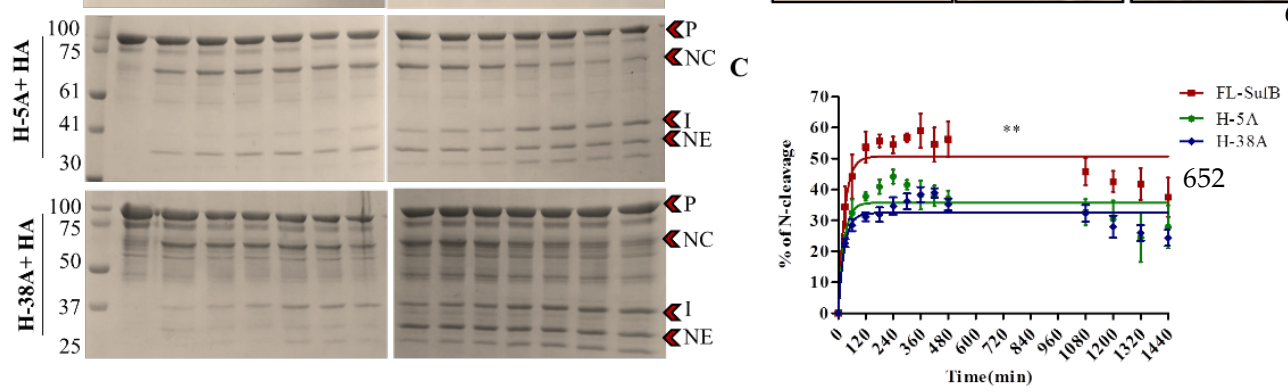

ઔ Possible precursor degradation product

- Off-pathway splicing intermediate

Figure 6. Effect of H-5A and H-38A mutation on HA mediated N-cleavage reaction in Mtu SufB : (A) SDS Gel showing HA induced N-cleavage reactions in (i) FL- SufB precursor (ii) H5A and (iii) H-38A SufB mutant; (B) Confirmation of splicing and cleavage products for HA induced N-cleavage reaction in (i) FL- SufB precursor, (ii) H-5A and (iii) H-38A mutant proteins via western blot. Anti-His antibodies detected presence of 6X (His) tagged P, CC and LE. NE is blotted separately with higher concentration of primary antibody; (C) (ii) Kinetic analysis of HA induced N-cleavage in WT and H-5A mutant proteins suggests statistically significant differences $(\mathrm{P}=0.0025$; one way ANOVA $)$ in $\%$ of $\mathrm{N}$-cleavage over different time periods. These curves were fitted into a pseudo first order reaction with equation $\mathrm{Y}=\mathrm{Y} 0+\left(\right.$ Plateau-Y0) $*\left[1-\exp ^{\left(-\mathrm{K}^{*}\right)}\right]$. All the experiments were performed in triplicates and error bars represent $( \pm 1) \mathrm{SEM}$. $\mathrm{P}=\mathrm{Precursor}, \mathrm{NC}=\mathrm{N}$ cleavage, $\mathrm{LE}=$ Ligated Extein, $\mathrm{I}=$ Intein, $\mathrm{NE}=\mathrm{N}$-Extein, $\mathrm{CC}=\mathrm{C}$-Cleavage. 
Table 2. (i) Comparative analysis of TCEP mediated $\mathrm{N}$-terminal cleavage reaction at $20^{\circ} \mathrm{C}$ over different time up to 240 mins. The data were extracted from Figure5B. (ii) Different kinetic parameters ( $\mathrm{Y}_{\max }$, rate constant, and half-life) for HA-induced N-terminal cleavage in FL-SufB precursor, H-5A, and H-38A SufB mutants. These data were extracted from Figure 6C.

(i)

\begin{tabular}{|c|c|c|c|}
\hline Time (min) & FL-SufB (\% of N-cleavage) & H-5A (\% of N-cleavage) & H-38A (\% of N-cleavage) \\
\hline $\mathbf{2 0}$ & $28.2 \pm 5.5$ & $15.1 \pm 0.97$ & $7.42 \pm 2$ \\
\hline $\mathbf{4 0}$ & $36 \pm 4.3$ & $17.89 \pm 2.7$ & $9.3 \pm 2.7$ \\
\hline $\mathbf{6 0}$ & $30.63 \pm 5.1$ & $20.76 \pm 3.7$ & $10.2 \pm 1.7$ \\
\hline $\mathbf{8 0}$ & $25.7 \pm 5.7$ & $14.9 \pm 2.5$ & $12.42 \pm 1.9$ \\
\hline $\mathbf{1 2 0}$ & $19.8 \pm 1.6$ & $8.4 \pm 0.7$ & $10.5 \pm 2.7$ \\
\hline $\mathbf{2 4 0}$ & $16.78 \pm 1.5$ & $6.2 \pm 0.89$ & $7.07 \pm 1.9$ \\
\hline
\end{tabular}

(ii)

\begin{tabular}{|c|c|c|c|}
\hline Kinetic parameters & FL-SufB & H-5A & H-38A \\
\hline $\mathbf{Y}_{\max }$ & $50.67 \pm 1.6$ & $35.72 \pm 1.4$ & $32.45 \pm 0.94$ \\
\hline $\begin{array}{c}\text { Rate Constant }(\mathbf{K}) \\
\left(\mathbf{m i n}^{-1}\right)\end{array}$ & $3.7 \pm 0.01 \times 10^{-2}$ & $3.9 \pm 0.01 \times 10^{-2}$ & $3.7 \pm 0.009 \times 10^{-2}$ \\
\hline Half-life(t) & $18.5 \mathrm{~min}$ & $17.3 \mathrm{~min}$ & $18.2 \mathrm{~min}$ \\
\hline
\end{tabular}




\subsection{Confirmation of protein products}

The identity of splicing and cleavage products (P, CC, LE, and NE) for the N-terminal 6X (His) tagged test proteins were confirmed via western blot using anti-His antibodies (Figure 3B, 4B, 6B and S4B). SI (C1A/N359A) double mutant SufB was used as a control for the study and gave rise to unspliced precursor protein (P) [Figure 3B (iv)]. Interestingly, one additional protein band was noticed above LE and below CC for FL- SufB, H-5A, and H-38A mutants possibly due to the generation of a splicing intermediate as an off-pathway product. We also observed a product just below the P protein for FL-SufB, SI-SufB, H-5A, and H-38A mutants (Figure3B, 6B, and S4B). However, this protein was missing in cells expressing SufB intein or empty vector pACYC Duet-1 [Figure 3B (iv)]. Hereby, we conclude that we are getting a degradation product of SufB precursor in the cells over-expressing active and inactive Mtu full-length (FL) SufB precursor protein.

Next, MALDI-TOF/TOF mass spectrometry was performed for further confirmation of different splicing and cleavage products (Table S3). Different protein products were cut from SDS PAGE gel and subjected to protein identification by mass spectrometry. First, acquired MS and MS/MS spectra were searched against the taxonomy Mycobacterium tuberculosis from NCBInr (77) database using mascot search engine in Protein Pilot Software. It is found that all the splicing products were identified as SufB protein of Mycobacterium tuberculosis complex with a statistically significant score. As all the splicing products were derivatives of a single protein, the protein identification results confirmed the same (Table S3). Further, acquired MS and MS/MS spectra were checked against a customized database containing probable splicing and cleavage products of SufB protein of Mycobacterium tuberculosis using mascot search engine in Protein Pilot Software with same parameters. 
The individual protein band spectra resulted in the identification of protein splicing products matching to it. Individual protein bands identification result is represented with protein score, percent coverage, and theoretical molecular weight and Isoelectric $\mathrm{pH}$ value in Table S3. Significance is measured from the expectancy-value (with a p-value $\leq 0.05$ ).

We performed HPLC analysis to find out fragment weight against a known protein ladder as mentioned in materials and methods. Protein fragments of Mtu FL-SufB were found to be within the expected mass range as per retention time (RT) from HPLC (Figure S1).

The molecular weight of SufB DM ( $\mathrm{H}-5 \mathrm{~A} / \mathrm{H}-38 \mathrm{~A})$ protein was determined by FPLC, as mentioned in the experimental section. The eluted fractions of both, BSA standard and DM protein were analyzed by SDS gel electrophoresis under both reducing and non-reducing conditions, yielding molecular weights of $66 \mathrm{KDa}$ and $28 \mathrm{KDa}$, respectively (Figure S3). No significant difference was observed in their molecular weights under reducing and non-reducing conditions, thereby concluding the existence of both the proteins in their monomeric form.

\subsection{Proposed mechanism for the cleavage of $\mathrm{N}$ - terminal intein $\sim$ extein peptide bond}

The current work investigates the mechanism of Mtu SufB intein cleavage at the N-terminal cleavage site between Gly252 (G-1) and Cys253 (C1). We have identified two highly conserved histidines at -5 (248, full-length protein) and -38 (215, full-length protein) positions of the Nextein sequence from different intein-carrying and intein-less bacterial and archeal SufB proteins. Our in vitro experiments suggested that His-5 (248) and His-38 (215) are likely to exert a regulatory role on SufB splicing and/or N-cleavage reactions.

To ascertain this hypothesis, we performed MD simulations to predict a 3-dimensional (3D) model for Mtu full-length (FL)-SufB precursor protein (Figure 7A). This also included 
735 identification of possible critical residues at the SufB intein active site (N- and C-terminal splice

736 junctions) (Figure 7B and Figure S8), given their proximity to catalytic residues Cys253 (C1),

737 Cys612 (C+1), and Asn611 (N359).

738

A

739

740

741

742

743

744

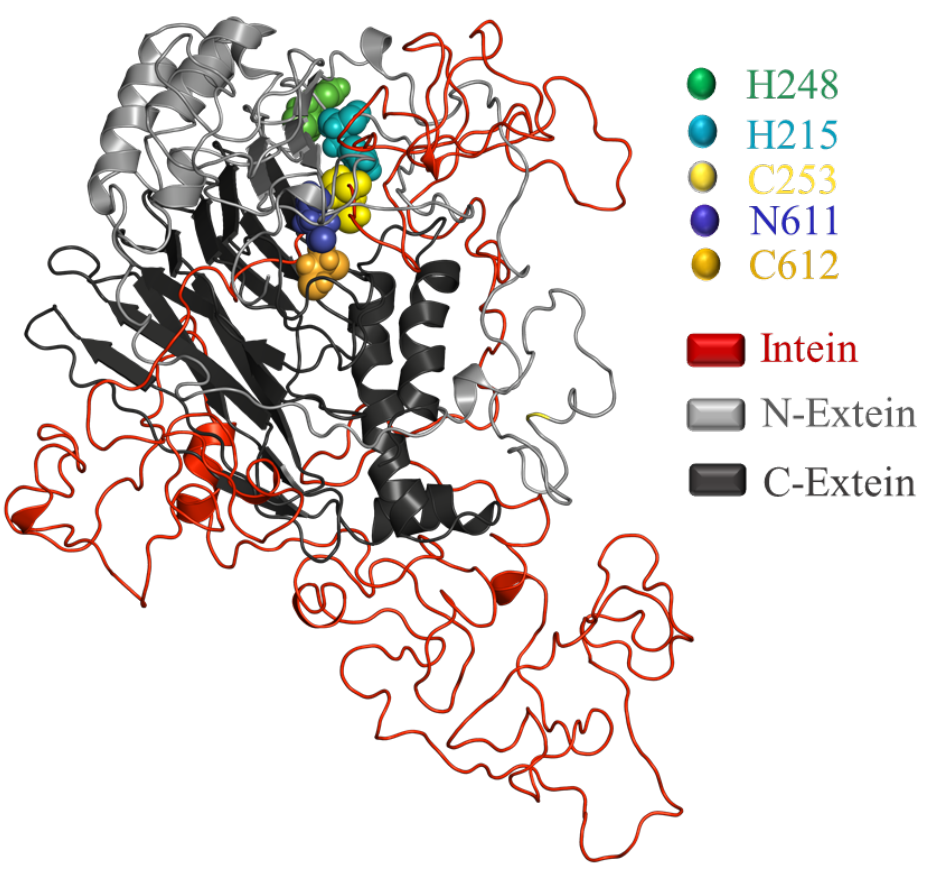

746

B

747

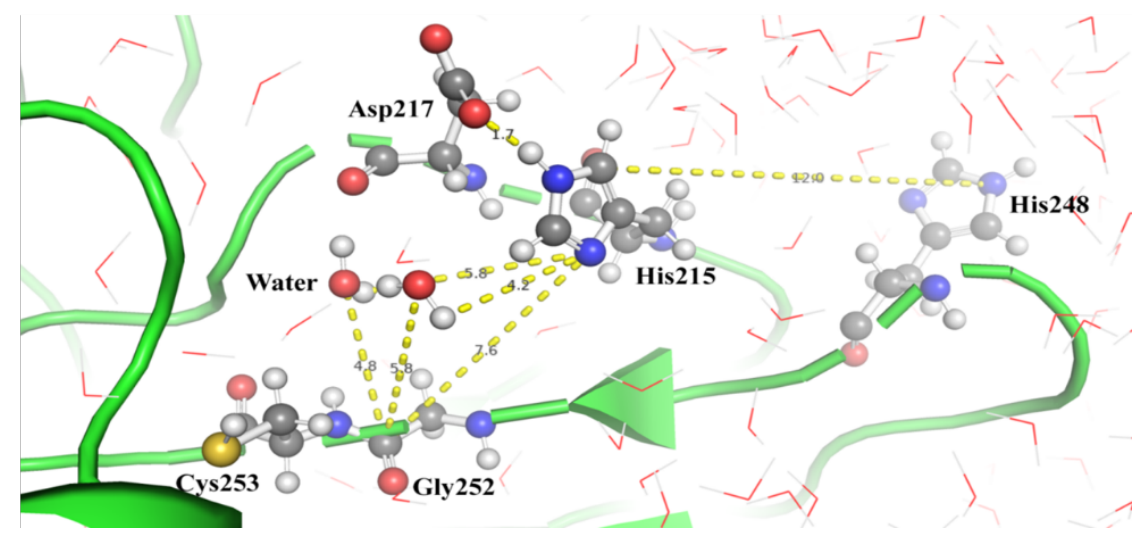


C<smiles>CCNC(=O)[C@H](CS)NC(=O)CNC(=O)CNC(=O)N[C@H](C(=O)O)C(=O)NC</smiles><smiles>CCCNC(=O)C(N)CSC(=O)CNC(C)=O</smiles><smiles></smiles>

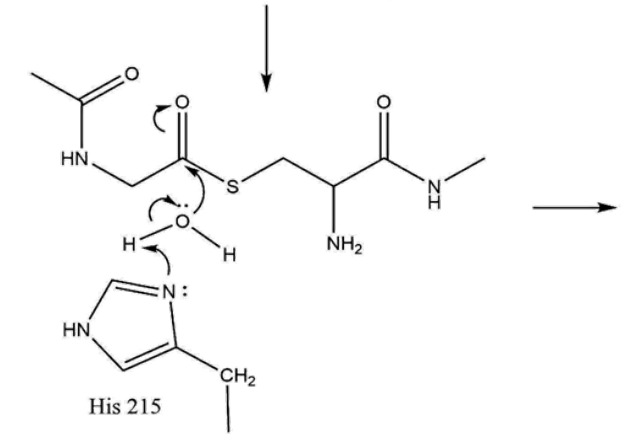<smiles>CNC(=O)C1CSC(CNC(C)=O)(C2(CNC(C)=O)CCO2)N1</smiles><smiles>CNC(=O)C(N)CSCC(O)(CNC(C)=O)C([O-])O</smiles><smiles>CNC(=O)C(N)CS</smiles>

766 


\section{D}

771

772

773

774

775

776

778

779

780

781

782

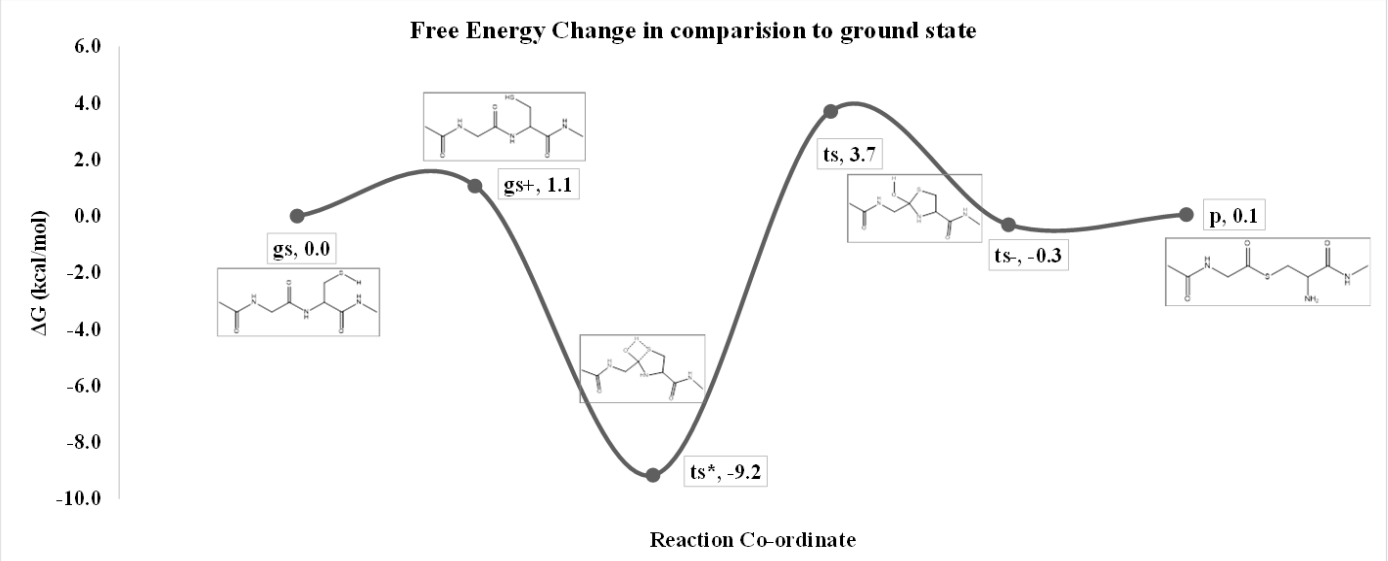

$\mathbf{E}$

777

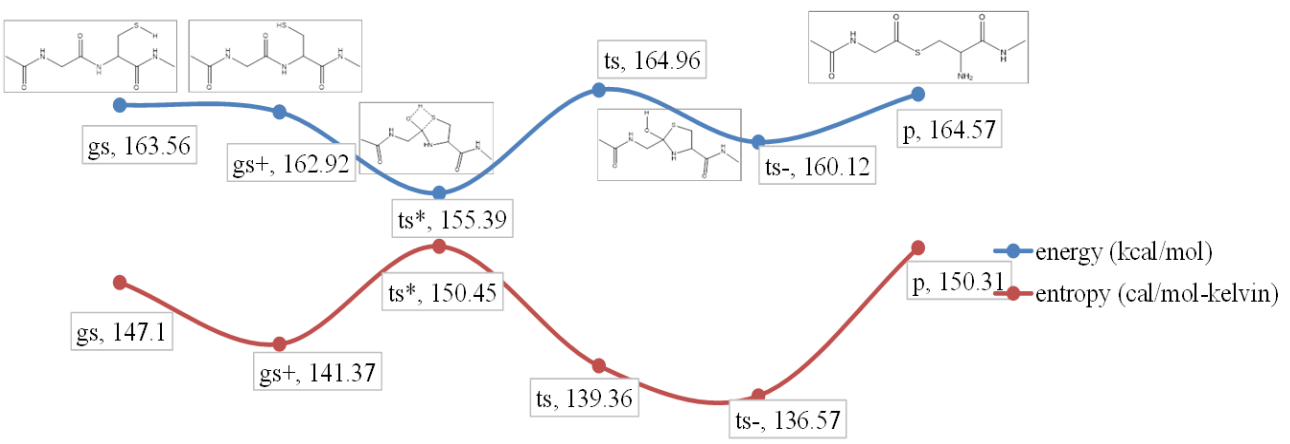

Reaction co-ordinate

Figure 7. Structural model and proposed mechanism for N-cleavage reaction in $M$ tu FLSufB precursor. (A) The 3D model of Mtu FL-SufB precursor obtained in presence of water and $\mathrm{NaCl}$. Different domains and conserved catalytic residues are color coded as shown in the legend, (B) Spatial arrangement of the N-terminal active site residues; Cys253 (C1) and Gly252 (G-1) along with His248 (H-5), His215 (H-38) and Asp217 (D-36) at the N-terminal cleavage site, $(\mathbf{C})$ Proposed mechanism for $\mathrm{N}$ - terminal cleavage reaction at intein $\sim$ exetin junction, (D) Free energy change, and (E) Energy and entropy of the system during QM calculation of different reaction states of N-cleavage junction from peptide bond to thio-ester bond of Mtu FL-SufB precursor protein. $\mathrm{gs}=$ ground state, $\mathrm{gs}+=$ conformational transition state, $\mathrm{ts}^{*}=$ transition state of proton transfer, $\mathrm{ts}=$ tetrahedral intermediate, $\mathrm{ts}=$ transition state of proton transfer, $\mathrm{p}=$ product. 
A chimera structure of Mtu FL-SufB protein was built in Schrodinger Bioluminate 2.7 using different templates based on their sequence identity with Mtu FL-SufB (Figure 7A). The sequence identity of Mtu FL-SufB to SufBCD complex from Escherichia coli (5AWF_A), PI-SceI; a homing endonuclease with protein splicing activity (1VDE_A) and intein homing endonuclease II (2CW7_A) was found to be $40 \%, 45 \%$, and $22 \%$ respectively. The resultant structure of Mtu FLSufB was found to have many loops in the intein region because of low sequence identity to the templates. In addition, nearly $13 \%$ of amino acids are found to be present in the disallowed region in the Ramachandran plot (Figure S6A). Therefore, we carried out molecular dynamics (MD) simulations of the aforementioned sub-optimized Mtu SufB structure in presence of water and $\mathrm{NaCl}$ to mimic in-vivo conditions in GROMACS for proper folding of the protein. The structure was solvated in water using the SPC/E water model $(59,60)$ and interactions were described through the OPLS-AA force field (58). The system was minimized by steepest descent minimization with a target maximum force not greater than $1000 \mathrm{~kJ} \mathrm{~mol}^{-1} \mathrm{~nm}$. The simulations were done using GROMACS with time steps of $2 \mathrm{fs}$ for a duration of $100 \mathrm{~ns}$.

After the 100ns simulation various parameters such as solvent accessible surface area (SASA) (Figure S5B), root mean square deviation (RMSD) was computed and the structure was found to be stabilized after 80ns, suggesting that the dynamics have reached equilibrium (Figure S5A). Models of Mtu FL-SufB and H-5A SufB mutant were simulated for 20ns. Their RMSD and Root Mean Square Fluctuation (RMSF) were compared (Figure S7A and B). The equilibrated Mtu SufB structure obtained from the above MD simulations was found to have only $\sim 1 \%$ amino acids in the disallowed region of the Ramachandran plot (Figure S6B). We found that the long side chains of the amino acids that were present in the disallowed region of the Ramachandran plot 
were exposed outside. The model was further validated by ProSA web before and after 100ns of MD simulation (Figure $\mathrm{S} 6 \mathrm{C}, \mathrm{D})$.

Next, the trajectory analysis of the Mtu FL-SufB structure was done to find out the possible role of critical residues including His248 (-5) present near the N- and C- cleavage sites. In particular, we followed the dynamics of His248 (-5) with respect to Gly252 (-1). Our MD simulations showed an interesting dynamical result. It was observed that His248 (-5) pushes another N-extein residue His215 (-38) towards the active site Gly252 (-1). In other words, the MD result suggests that His248 (-5) and His215 (-38) act in a concerted manner to facilitate Mtu SufB N-cleavage reaction. We should point out here, although His248 (-5) is closer to the active site compared to His215 (-38) in the primary sequence, our 3-dimensional structure finds the opposite i. e. His 215 (-38) is closer to the active site relative to His248 (-5). Specifically, the shortest dynamical distance between His215 (-38) and Gly252 (-1) was observed to be $6.83 \AA$, similar to catalytic distances observed between active residues in other intein systems $(12,34)$. Therefore, we propose that His248 (-5) and His215 (-38) act concordantly to facilitate the Mtu SufB N-cleavage reaction as explained in the discussion section.

Based on the above MD results, a schematic cleavage reaction is shown in (Figure 7C) along the line of canonical intein splicing mechanism (78). Thus, we propose that conjoint effects of active site (N-terminal intein extein splice junction) residues such as Gly252 (-1), Cys253 (1), and His215 (-38) supported by structural and conformational changes due to His248 (-5) contribute towards Mtu SufB N-terminal cleavage reaction. To provide further confidence to our structural and dynamical data, quantum mechanical (QM) calculations were required to obtain energetics of $\mathrm{N}$-cleavage reaction and compared with earlier results. All the quantum chemical calculations were performed with the Gaussian16 package $(32,79,80)$. All geometry optimization of the 
molecules was computed using the B3LYP method of DFT theory at 6-31G (d) level with chargeneutral singlet $(32,79,81)$. Similar techniques were used in the study of intein cleavage of Mtu Rec Intein (17). After the optimized structures of the reactants and the products for each step were obtained, they could be used as the initial and final states respectively in the TS module to find the corresponding energy barriers. Frequency analyses were performed at the same level to identify all the stationary points of the intermediates (only real frequencies) and the saddle points of the transition states (one imaginary frequency). The reaction energy barrier was obtained from the energy difference between the transition state and reactant of the reaction with the zero-point correction. All stationary states were confirmed by means of vibrational frequency analysis, and the Gibbs free energy at physiological temperature was calculated for all stationary points, including the zero-point energy, the entropy, and the thermal energy (all at 298.15K) at the B3LYP/6-31G (d) (Figure 7D, 7E). After exploring the reaction energy landscape and obtaining the optimized geometries for intermediates, transition states, and products, we hereby propose a detailed reaction mechanism for Mtu full-length SufB N-cleavage reaction as elucidated in the discussion section (Figure 7C).

Further, the amino acids near the C-cleavage site in the 3D model of Mtu SufB are identified as His610 (358), Asn 611 (359), and Cys612 (+1) (Figure S8). The distance between Asn611 (N359)

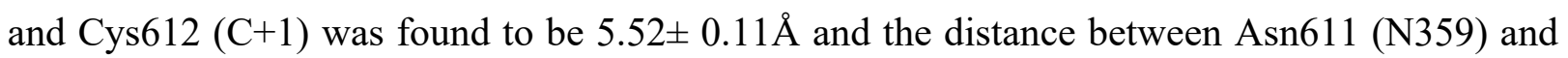
His610 (H358) was $4.33 \pm 0.25 \AA$ (Table S5). Due to their proximity, the C-cleavage reaction may be facilitated by interactions between these three amino acids. Future research could shed light on the possible roles of these residues in the mechanism of C-cleavage reaction for Mtu FL- SufB. 


\section{DISCUSSION}

\subsection{Mtu SufB harbors a classic intein splicing system with two conserved histidine residues in the $N$-extein region}

SufB is the central and essential component of the mycobacterial SUF system. This work reports that SufB protein is well conserved in mycobacteria, bacteria, and archaea during the evolutionary course. Intein insertion points may vary in some species, but the conservation of critical residues in intein and extein regions does exist. Intein-carrying SufB proteins are found in different mycobacterial species (Figure 2) (51). Simple sequence analysis depicted the conservation of SufB intein in different organisms and phylogenetic analysis of intein and whole sequence inferred the possibility of common ancestor through evolution (Figure 2). Cladogram analysis suggested a trivial diversion in M. leprae, M. lepromatosis, M. xenopi, M. triplex and Ferroplasma. The discrepancies in the phylogenetic tree analyses of un-spliced SufB precursors indicated that there are diversity in the extein sequences and intein insertion sites. This suggests intein evolution and independent intein transfer in different species and kingdoms.

Domain analysis of Mtu SufB precursor; an 846 aa residue protein clearly demarcated the intein and extein structural regions (Figure 1, Table S2). We found that Mtu SufB intein spanning over 359 residues has an intact endonuclease domain. Catalytic residues critical for classic intein splicing pathways like Cys1 (Block A), Cys+1 (Block G), penultimate His (Block G), and terminal Asn (Block G) are conserved in different mycobacterial species including Mtu. Besides, Mtu SufB intein also contains conserved Block B His67 that is known to catalyze the first N-S acyl shift by destabilizing the scissile peptide bond due to loss of resonance and reduction in energy barrier. In vitro refolding of Mtu SufB precursor gave rise to splicing and cleavage 
products confirming the catalytic roles of the aforementioned conserved residues. Thus, we conclude that Mtu SufB carries a canonical (classic) cis- splicing intein system (Figure 1). Furthermore, sequence analysis also detected two highly conserved His residues in the N-extein sequence of Mtu SufB protein; His-38 and His-5 located in all SufB proteins where SUF constitutes the exclusive pathway for [Fe-S] cluster generation irrespective of genus and kingdoms (Figure 2A, B). Later, these histidines were identified to play key regulatory roles during Mtu SufB N-cleavage reaction.

\subsection{A distinct extein intein partnership mechanism guided by highly conserved $\mathrm{H}-5$ and $\mathrm{H}-38$} regulates Mtu SufB $N$-cleavage reaction

Catalytic residues such as Cys1, Cys +1 , and terminal Asn directly participate in intein splicing via promoting sequential nucleophilic displacement reactions or by rearrangement of bonds near splice junctions. Non-catalytic residues assist indirectly via activation of active site residues and stabilization of various intermediate structure(s). Conserved His residues within intein sequence are known to play important roles during protein splicing as well. Block B His accelerates N-S acyl shift and cleavage of N-terminal intein $\sim$ extein peptide bond whereas F- and G-Block His are crucial in the coordination of terminal Asn cyclization and cleavage of C-terminal splice site. We found that Mtu SufB displays a unique mechanism where two remote histidines located in the Nextein sequence work together to facilitate N-cleavage reaction via activation of catalytic Cys1.

To ascertain the roles of H-5 and H-38, a detailed kinetic analysis was performed on Mtu SufB full-length (FL) precursor and mutant proteins (H-5A and H-38A) [Figure 3, 4, and 5]. We found that both $\mathrm{H}-5 \mathrm{~A}$ and $\mathrm{H}-38 \mathrm{~A}$ mutants exhibit a sluggish splicing reaction relative to FL- SufB protein under optimum experimental conditions (Table 1). N-cleavage reaction kinetics of the 
aforesaid histidine mutants were examined in presence of TCEP and nucleophiles such as Hydroxylamine and DTT. H-5A and H-38A mutants demonstrated a significant reduction in Ncleavage efficiency in comparison to FL- SufB protein in presence of TCEP and nucleophile HA (Table 2 (i) and (ii), Figure5 and 6). But DTT-induced thiolysis reversed the effect of alanine mutation in the histidine mutants. DTT is a thiol protectant reducing agent and exhibits a more efficient thiolysis and di-sulfide reduction. A strong nucleophile such as DTT abolishes the inhibitory effect of alanine mutation on SufB N-cleavage reaction. Herein, we may conclude that H-5 and H-38 possibly have an assistive role in Mtu SufB N-cleavage reaction along with other active site residues. Consequently, the effect of $\mathrm{H}-5 \mathrm{~A}$ and $\mathrm{H}-38 \mathrm{~A}$ mutation can be reversed in presence of strong nucleophile(s) like DTT (Figure S4).

To further ascertain the roles of conserved histidines, a 3D model of the Mtu FL-SufB protein was built by homology modeling. Molecular dynamics (MD) simulations were performed to equilibrate the aforementioned sub-optimized Mtu SufB structure. We obtained an interesting result during the trajectory analysis of the dynamical data. Although $\mathrm{H}-38$ is relatively distal to the N-terminal splice junction in the protein sequence, in the 3-dimensional structure, it comes closer to the active site. MD results suggest that His248 (-5) and His215 (-38) act in a concerted manner to facilitate $\mathrm{Mtu}$ SufB N-cleavage reaction. Thus, we propose that concomitant effects of N-terminal active site residues such as Gly252 (-1), Cys253 (1), and His215 (-38) supported by His248 (-5) contribute towards Mtu SufB N-terminal cleavage reaction. Next, QM/MM calculations were done to determine the energetics of the proposed N-cleavage reaction. Taken together, we hereby propose a detailed mechanism for Mtu SufB N-cleavage reaction. A schematic for the same is depicted in (Figure 7C) along the line of canonical intein splicing mechanism. 
In the process to form thioester intermediate from peptide bond of Gly-Cys dipeptide capped with a methyl group, the steps are as follows. The thiol group of Cys253 (1) comes closer to peptidyl $\mathrm{C}=\mathrm{O}$ by a conformational change to a rotational transition state. This state is enthalpy favored. Then the thiol $\mathrm{H}$ is polarized towards the peptidyl $\mathrm{O}$ atom. The $\mathrm{C}=\mathrm{O}$ and $\mathrm{S}-\mathrm{H}$ experiences change in charge density in the system. The $\mathrm{C}, \mathrm{S}, \mathrm{H}$ atoms in the system experience decrease in electron density, and the $\mathrm{O}$ atom experiences an increase in electron density. In the next transition state, $\mathrm{C}$ and $\mathrm{S}$ come closer as at this state the imaginary vibrational frequency displacement vector of $\mathrm{C}=\mathrm{O}$ is towards thiol. Thus, a C-S bond is formed. The proton migration from $\mathrm{S}$ to $\mathrm{O}$ is highly favored energetically and entropically with a free energy change of $-9.2 \mathrm{kcal} / \mathrm{mol}$. The computed value agrees well with earlier results $(82,83)$. After this step $\mathrm{sp}^{2}$ hybridized peptide carbon becomes $\mathrm{sp}^{3}$ hybridized forming a tetrahedral transition state, a 2-hydroxy thiazolidine ring. This state annihilates the peptide resonance, therefore free energy change of $+3.7 \mathrm{kcal} / \mathrm{mol}$ in contrast to the ground state of Gly252 (-1) and Cys253 (1) system. To again regain the $\mathrm{sp}^{2}$ hybridization and decrease the energy of the system from resonance stabilization, the 2-hydroxy group expels proton to the environment. This process is energetically advantageous to the system elucidating negative free energy. In a tetrahedral state, $\mathrm{S}$ and $\mathrm{N}$ atoms have a Mulliken charge of +0.05 and -0.55 . Thus, the $\mathrm{N}$ atom of the thiazolidine ring accepts a proton from the environment to the system for charge neutrality. This process is also entropically preferred to form thioester intermediate $(82,84)$. The conversion of a peptide bond to a thioester bond is energetically equivalent at the start as well as the end of the reaction which suggests this process is entropy driven. Further thioester is hydrolyzed by the imidazole side chain of His215 (-38) mediated by water. Base catalyzed thioester hydrolysis in an aqueous environment is well known (85). It is also important to point out that the least distance between His215 (-38) and His248 (-5) obtained 
in $\mathrm{MD}$ simulations after equilibrium is about $6.83 \AA$ suggesting catalytic distance for an interaction between these two residues $(12,13)$. Asp217 (-36) prepares protonated histidine His215 (-38) for catalyzing reactions by neutralizing charge through proton transfer. Furthermore, His248 (-5) which is exposed to the solvent, experiences random collisions from water molecules. This action forces His215 (-38) towards the N-cleavage site. This is corroborated by the dynamical distance pattern between His215 (-38) and His248 (-5). Thus, a congruent effort by His248 (-5) facilitates the thioester hydrolysis for cleavage by attacking species i.e., hydroxide ion generation catalyzed by His215 (-38). These results provide confidence in the proposed role of His248 (-5) and His215 (-38). Since histidines are known as metal chelating centers for various metalloproteins, conserved histidines at -5 and -38 positions might coordinate to [Fe-S] clusters contributing towards Mtu SufB functionality as well.

\subsection{Biological significance of conserved histidines in the N-extein sequence of Mtu SufB}

[Fe-S] cluster-bearing proteins have important physiological roles in electron transfer, redox regulation, metabolic pathways, cellular responses to external stimuli, and as regulators of gene expression (42). The role of the [Fe-S] cluster is closely associated with the functionality of their bound protein framework. In mycobacteria, the SUF system is the sole pathway for [Fe-S] cluster assembly and repair, especially in response to oxidative stress and iron limiting conditions inside macrophages $(43,49)$. During such an event, the intracellular Fe supply is either from siderophore chelation or via the metabolism of [Fe-S] clusters associated with specific proteins (42). Thus, iron homeostasis plays a major role in mycobacterial survival and virulence. SufB is a $[\mathrm{Fe}-\mathrm{S}]$ cluster scaffold protein and a vital component of the functional SUF system, and indirectly promotes mycobacterial persistence under stress (49). Further, SufB has been implicated in mycobacterial iron metabolism (42). 
973 Earlier studies have specified the bonding of Fe in [Fe-S] clusters to mostly cysteines from the

974

975

976

977

978

979 protein backbone although there is increasing evidence for other ligands such as histidine, aspartate, arginine, threonine, and tyrosine. The most common alternative ligand for [Fe-S] cluster coordination is histidine that is highly conserved with a role in redox tuning and protoncoupled electron transfer (86). His433 (SufB) and His360 (SufD) are identified as key proteinligands for the de novo [Fe-S] cluster assembly in E. coli (48). It has been shown that these noncysteine ligands can influence the stability and reactivity of [Fe-S] clusters.

We have identified two highly conserved His-5 and His-38 residues in the N-extein sequence of $M t u$ SufB. These were shown to interact concordantly to initiate N-cleavage reaction via catalytic Cys1 activation. Mutational analysis of alanine-substituted His-5 and His-38 residues revealed significantly attenuated cleavage and splicing rates. Attempts to express and analyze the Mtu SufB double mutant (His-5A/ His-38A) resulted in a truncated protein product. One of the possible explanations could be the concomitant role of His-5 and His-38 in SufB precursor stabilization. Lack of the histidine residues at the native position perhaps leads to loss of protein stability forming off-pathway splicing intermediate and/or protein degradation product. In summary, the two histidines (His-5 and His-38) work concurrently to activate/initiate N-cleavage reaction leading to the generation of functional SufB protein via protein splicing. As proposed by earlier studies, apart from Cys, His is also an important ligand for metal coordination $(48,86)$. Thus, it is plausible that the highly conserved His-5 and His-38 possibly coordinate to $\mathrm{Fe}^{+2} / \mathrm{Fe}^{+3}$ during [Fe-S] cluster biogenesis. Lastly, His-5 and His-38 together might also play a role in Mtu SufB precursor stabilization prior to cleavage and splicing as we were unable to express a fulllength SufB double mutant (His-5A and His-38A), unlike the single mutants. However, whether 
the SufB precursor stabilization is executed by coordination of conserved histidines to [Fe-S] cluster or bonding to $\mathrm{Fe}^{+2} / \mathrm{Fe}^{+3}$, it needs extensive experimental and computational confirmation.

Suf is an exclusive system for biogenesis of Fe-S clusters in many pathogenic organisms such as Staphylococcus aureus, Mycobacterium tuberculosis, Plasmodium sp. and Toxoplasma, making it an attractive drug target. For instance, D-Cycloserine is a clinical second-line drug currently used against M. tuberculosis and inhibits SufS. Investigation on S. aureus revealed that SUF is the target system for a polycyclic molecule 882 that has direct interaction with SufC (69). Likewise, the current work may have an implication towards the development of novel anti-TB drugs targeting SufB destabilization, to fight tuberculosis.

Data Availability: All the data are available in the manuscript and supplemental data.

The accession code for the Mycobacterium tuberculosis SufB protein used in this study is $P 9 W F P 7$ (UniProtKB/Swiss-Prot) and WP_003407484.1, GI: 397673309 (NCBI protein database).

Confirmation of the identity of different splicing and cleavage products of Mtu FL-SufB protein by mass spectrometric analysis: The data has been deposited to the ProteomeXchange Consortium via PRIDE partner repository with data set identifier PXD015199. The details of the submission are given below.

Project Name: Identification of full length, splicing and cleavage products of SufB protein of SUF-complex of Mycobacterium tuberculosis.

Project accession: PXD015199

\section{Reviewer account details:}

Username- reviewer73867@ebi.ac.uk

Password: bRxRcbvP

Confirmation of the identity of H-38A (215)/H-5A (248) Mtu SufB double mutant product by mass spectrometric analvsis: The data has been deposited to the ProteomeXchange Consortium 

via PRIDE partner repository with data set identifier PXD023785. The details of the project submission are given below.

Project Name: Identification of splicing and cleavage products of H-38A/H-5A SufB double mutant protein of SUF-complex of Mycobacterium tuberculosis.

Project accession: PXD023785

\section{Reviewer account details:}

Username- reviewer_pxd023785@ebi.ac.uk

Password: 1i6COWkr

The homology model for the full length Mtu SufB protein has been submitted to Model Archive [Project: ma-x807d]:

Full length Mtu SufB protein of SUF complex of Mycobacterium tuberculosis; [https://www.modelarchive.org/doi/10.5452/ma-X807d] with the access code: 6pmXRNkvwR.

Funding: Current work and Ananya Nanda are supported by UGC-DAE consortium for scientific research(UGC-DAE-CSR-KC/CRS/15/IOP/08/0562), Kolkata, India. Dr. Sunita Panda (201500000557/IF 140155) was supported by INSPIRE fellowship; INSPIRE Division, DST, Government of India.

Acknowledgements: Our sincere thanks to Prof. Marlene Belfort, Department of Biological Sciences and RNA Institute, University at Albany, Albany, NY, USA, for her valuable comments on the manuscript. Plasmids used in this work were engineered by Dr. Sasmita Nayak during her PhD work under the guidance of Prof. Marlene Belfort. We also thank Mr. Sourya Subhra Nasker for providing critical comments on the manuscript. We are thankful to Mr. Rajendra Reddy for technical support at Central Proteomics Facility, Institute of Life sciences, and Bhubaneswar. We also thank Mr. A. Ananda Raman for his timely help in HPC (High Performance computing cluster) to perform computational work at National Institute of Science Education and Research 
(NISER) Bhubaneswar. Our sincere gratitude to Mrs. Anuradha Das for her help in conducting the HPLC experiment at School of Chemical Sciences, NISER Bhubaneswar and to Dr. Rahul Modak for his help in conducting FPLC at School of Biotechnology, KIIT Deemed to be University.

Conflict of Interest: Authors declare no conflict of interest.

Author contributions: Dr. Sunita Panda: Methodology; Validation; Formal analysis; Investigation; Resources; Data curation; Writing - original draft; Visualization; Funding acquisition. Ananya Nanda: Methodology; Validation; Formal analysis; Investigation; Resources; Data curation; Writing - original draft; Writing- Reviewing and Editing; Visualization. Nilanjan Sahu: Methodology; Software; Validation; Formal analysis; Writing original draft. Deepak Kumar Ojha: Validation; Formal analysis; Investigation; Resources. Dr. Biswaranjan Pradhan: Formal analysis; Resources. Anjali Rai: Resources. Dr. Amol R. Suryawanshi: Methodology; Validation. Dr. Nilesh Banavali: Methodology; Software; Validation; Writing- Reviewing and Editing; Visualization. Dr. Sasmita Nayak: Conceptualization; Methodology; Validation; Investigation; Data curation; Writing - original draft; Writing- Reviewing and Editing; Visualization; Supervision; Funding acquisition; Project administration.

\section{References}

1. Derbyshire V, Belfort M. Lightning strikes twice: intron-intein coincidence. Proceedings of the National Academy of Sciences. 1998;95(4):1356-7.

2. $\quad$ Lennon CW, Belfort M. Inteins. Current Biology. 2017;27(6):R204-R6. 3. Starokadomskyy P. Protein splicing. Molecular Biology. 2007;41(2):278-93.

4. Novikova O, Topilina N, Belfort M. Enigmatic distribution, evolution, and function of inteins. Journal of Biological Chemistry. 2014;289(21):14490-7.

5. Elleuche S, Pöggeler S. Inteins, valuable genetic elements in molecular biology and biotechnology. Applied Microbiology and Biotechnology. 2010;87(2):479-89.

6. Lennon CW, Stanger M, Banavali NK, Belfort M. Conditional Protein Splicing Switch in Hyperthermophiles through an Intein-Extein Partnership. mBio. 2018;9(1):e02304-17. 
7. Lennon CW, Stanger MJ, Belfort M. Mechanism of single-stranded DNA activation of recombinase intein splicing. Biochemistry. 2019;58(31):3335-9.

8. Nanda A, Nasker SS, Mehra A, Panda S, Nayak S. Inteins in Science: Evolution to Application. Microorganisms. 2020;8(12):2004.

9. Panda S, Nanda A, Nasker SS, Sen D, Mehra A, Nayak S. Metal effect on intein splicing: A review. Biochimie. 2021.

10. Lennon CW, Stanger M, Belfort M. Protein splicing of a recombinase intein induced by ssDNA and DNA damage. Genes \& development. 2016;30(24):2663-8.

11. Kelley DS, Lennon CW, Li Z, Miller MR, Banavali NK, Li H, et al. Mycobacterial DnaB helicase intein as oxidative stress sensor. Nature communications. 2018;9(1):1-15.

12. Eryilmaz E, Shah NH, Muir TW, Cowburn D. Structural and dynamical features of inteins and implications on protein splicing. Journal of Biological Chemistry. 2014;289(21):14506-11.

13. Hiraga K, Soga I, Dansereau JT, Pereira B, Derbyshire V, Du Z, et al. Selection and structure of hyperactive inteins: peripheral changes relayed to the catalytic center. Journal of molecular biology. 2009;393(5):1106-17.

14. Binschik J, Mootz HD. Chemical Bypass of Intein-Catalyzed N-S Acyl Shift in Protein Splicing. Angewandte Chemie International Edition. 2013;52(15):4260-4.

15. Du Z, Shemella PT, Liu Y, McCallum SA, Pereira B, Nayak SK, et al. Highly Conserved Histidine Plays a Dual Catalytic Role in Protein Splicing: A p K a Shift Mechanism. Journal of the American Chemical Society. 2009;131(32):11581-9.

16. Du Z, Zheng Y, Patterson M, Liu Y, Wang C. p K a Coupling at the Intein Active Site: Implications for the Coordination Mechanism of Protein Splicing with a Conserved Aspartate. Journal of the American Chemical Society. 2011;133(26):10275-82.

17. Friedel K, Popp MA, Matern JC, Gazdag EM, Thiel IV, Volkmann G, et al. A functional interplay between intein and extein sequences in protein splicing compensates for the essential block B histidine. Chemical science. 2019;10(1):239-51.

18. Mujika J, Lopez X. Unveiling the Catalytic Role of B-Block Histidine in the N-S Acyl Shift Step of Protein Splicing. The Journal of Physical Chemistry B. 2017;121(33):7786-96.

19. Pereira B, Shemella PT, Amitai G, Belfort G, Nayak SK, Belfort M. Spontaneous proton transfer to a conserved intein residue determines on-pathway protein splicing. Journal of molecular biology. 2011;406(3):430-42.

20. Ding Y, Xu M-Q, Ghosh I, Chen X, Ferrandon S, Lesage G, et al. Crystal structure of a mini-intein reveals a conserved catalytic module involved in side chain cyclization of asparagine during protein splicing. Journal of Biological Chemistry. 2003;278(40):39133-42.

21. Klabunde T, Sharma S, Telenti A, Jacobs WR, Sacchettini JC. Crystal structure of GyrA intein from Mycobacterium xenopi reveals structural basis of protein splicing. Nature structural biology. 1998;5(1):31-6.

22. Frutos S, Goger M, Giovani B, Cowburn D, Muir TW. Branched intermediate formation stimulates peptide bond cleavage in protein splicing. Nature chemical biology. 2010;6(7):527-33. 23. Mujika JI, Lopez X, Mulholland AJ. Modeling protein splicing: reaction pathway for Cterminal splice and intein scission. The journal of physical chemistry B. 2009;113(16):5607-16. 24. Shao Y, PAULUS E. Protein splicing: estimation of the rate of O-N and S-N acyl rearrangements, the last step of the splicing process. The Journal of peptide research. 1997;50(3):193-8. 
25. Johnson MA, Southworth MW, Herrmann T, Brace L, Perler FB, Wüthrich K. NMR structure of a KlbA intein precursor from Methanococcus jannaschii. Protein Science. 2007;16(7):1316-28.

26. Southworth MW, Benner J, Perler FB. An alternative protein splicing mechanism for inteins lacking an N-terminal nucleophile. The EMBO Journal. 2000;19(18):5019-26.

27. Brace LE, Southworth MW, Tori K, Cushing ML, Perler F. The Deinococcus radiodurans Snf2 intein caught in the act: detection of the Class 3 intein signature Block F branched intermediate. Protein Science. 2010;19(8):1525-33.

28. Tori K, Dassa B, Johnson MA, Southworth MW, Brace LE, Ishino Y, et al. Splicing of the mycobacteriophage Bethlehem DnaB intein: identification of a new mechanistic class of inteins that contain an obligate block F nucleophile. Journal of Biological Chemistry. 2010;285(4):251526.

29. Tori K, Perler FB. Expanding the definition of class 3 inteins and their proposed phage origin. Journal of bacteriology. 2011;193(8):2035-41.

30. Amitai G, Callahan BP, Stanger MJ, Belfort G, Belfort M. Modulation of intein activity by its neighboring extein substrates. Proceedings of the National Academy of Sciences. 2009;106(27):11005-10.

31. Shah NH, Eryilmaz E, Cowburn D, Muir TW. Extein residues play an intimate role in the rate-limiting step of protein trans-splicing. Journal of the American Chemical Society. 2013;135(15):5839-47.

32. Shemella P, Pereira B, Zhang Y, Van Roey P, Belfort G, Garde S, et al. Mechanism for intein C-terminal cleavage: a proposal from quantum mechanical calculations. Biophysical journal. 2007;92(3):847-53.

33. Sun P, Ye S, Ferrandon S, Evans TC, Xu M-Q, Rao Z. Crystal structures of an intein from the split dnaE gene of Synechocystis sp. PCC6803 reveal the catalytic model without the penultimate histidine and the mechanism of zinc ion inhibition of protein splicing. Journal of molecular biology. 2005;353(5):1093-105.

34. Topilina NI, Novikova O, Stanger M, Banavali NK, Belfort M. Post-translational environmental switch of RadA activity by extein-intein interactions in protein splicing. Nucleic acids research. 2015;43(13):6631-48.

35. Cheriyan M, Pedamallu CS, Tori K, Perler F. Faster protein splicing with the Nostoc punctiforme DnaE intein using non-native extein residues. Journal of Biological Chemistry. 2013;288(9):6202-11.

36. Chong S, Williams KS, Wotkowicz C, Xu M-Q. Modulation of protein splicing of the Saccharomyces cerevisiae vacuolar membrane ATPase intein. Journal of Biological Chemistry. 1998;273(17):10567-77.

37. Oeemig JS, Zhou D, Kajander T, Wlodawer A, Iwaï H. NMR and crystal structures of the Pyrococcus horikoshii RadA intein guide a strategy for engineering a highly efficient and promiscuous intein. Journal of molecular biology. 2012;421(1):85-99.

38. Shemella PT, Topilina NI, Soga I, Pereira B, Belfort G, Belfort M, et al. Electronic structure of neighboring extein residue modulates intein C-terminal cleavage activity. Biophysical journal. 2011;100(9):2217-25.

39. Topilina NI, Green CM, Jayachandran P, Kelley DS, Stanger MJ, Piazza CL, et al. SufB intein of Mycobacterium tuberculosis as a sensor for oxidative and nitrosative stresses. Proceedings of the National Academy of Sciences. 2015;112(33):10348-53. 
40. Mills KV, Johnson MA, Perler FB. Protein splicing: how inteins escape from precursor proteins. Journal of Biological Chemistry. 2014;289(21):14498-505.

41. Cheriyan M, Chan S-H, Perler F. Traceless splicing enabled by substrate-induced activation of the Nostoc punctiforme Npu DnaE intein after mutation of a catalytic cysteine to serine. Journal of molecular biology. 2014;426(24):4018-29.

42. Huet G, Daffé M, Saves I. Identification of the Mycobacterium tuberculosis SUF machinery as the exclusive mycobacterial system of [Fe-S] cluster assembly: evidence for its implication in the pathogen's survival. Journal of bacteriology. 2005;187(17):6137-46.

43. Outten FW. Recent advances in the Suf Fe-S cluster biogenesis pathway: beyond the Proteobacteria. Biochimica et Biophysica Acta (BBA)-Molecular Cell Research. 2015;1853(6):1464-9.

44. $\quad$ Lin C-W, McCabe JW, Russell DH, Barondeau DP. Molecular Mechanism of ISC IronSulfur Cluster Biogenesis Revealed by High-Resolution Native Mass Spectrometry. Journal of the American Chemical Society. 2020;142(13):6018-29.

45. Mettert EL, Kiley PJ. Fe-S proteins that regulate gene expression. Biochimica et Biophysica Acta (BBA)-Molecular Cell Research. 2015;1853(6):1284-93.

46. Novikova O, Jayachandran P, Kelley DS, Morton Z, Merwin S, Topilina NI, et al. Intein clustering suggests functional importance in different domains of life. Molecular biology and evolution. 2016;33(3):783-99.

47. Outten FW, Djaman O, Storz G. A suf operon requirement for Fe-S cluster assembly during iron starvation in Escherichia coli. Molecular microbiology. 2004;52(3):861-72.

48. Yuda E, Tanaka N, Fujishiro T, Yokoyama N, Hirabayashi K, Fukuyama K, et al. Mapping the key residues of SufB and SufD essential for biosynthesis of iron-sulfur clusters. Scientific reports. 2017;7(1):9387.

49. Huet G, Castaing J-P, Fournier D, Daffé M, Saves I. Protein splicing of SufB is crucial for the functionality of the Mycobacterium tuberculosis SUF machinery. Journal of bacteriology. 2006;188(9):3412-4.

50. Larking M, Blackshields G, Brown N, Chenna R, McGettigan G, McWilliam H, et al. ClustalW and ClustalX version 2. Bioinformatics. 2007;23(21):2947-8.

51. Perler FB. InBase: the intein database. Nucleic acids research. 2002;30(1):383-4.

52. Morgenstern B. DIALIGN 2: improvement of the segment-to-segment approach to multiple sequence alignment. Bioinformatics (Oxford, England). 1999;15(3):211-8.

53. Kumar S, Stecher G, Li M, Knyaz C, Tamura K. MEGA X: molecular evolutionary genetics analysis across computing platforms. Molecular biology and evolution. 2018;35(6):15479.

54. Truszkowski J, Goldman N. Maximum likelihood phylogenetic inference is consistent on multiple sequence alignments, with or without gaps. Systematic biology. 2016;65(2):328-33.

55. Mills KV, Paulus H. Reversible inhibition of protein splicing by zinc ion. Journal of Biological Chemistry. 2001;276(14):10832-8.

56. Wiederstein M, Sippl MJ. ProSA-web: interactive web service for the recognition of errors in three-dimensional structures of proteins. Nucleic acids research. 2007;35(suppl_2):W407-W10. 57. Abraham MJ, Murtola T, Schulz R, Páll S, Smith JC, Hess B, et al. GROMACS: High performance molecular simulations through multi-level parallelism from laptops to supercomputers. SoftwareX. 2015;1:19-25. 
58. Kaminski GA, Friesner RA, Tirado-Rives J, Jorgensen WL. Evaluation and reparametrization of the OPLS-AA force field for proteins via comparison with accurate quantum chemical calculations on peptides. The Journal of Physical Chemistry B. 2001;105(28):6474-87. 59. Berendsen H, Grigera J, Straatsma T. The missing term in effective pair potentials. Journal of Physical Chemistry. 1987;91(24):6269-71.

60. Kusalik PG, Svishchev IM. The spatial structure in liquid water. Science. 1994;265(5176):1219-21.

61. Fletcher R, Powell MJ. A rapidly convergent descent method for minimization. The computer journal. 1963;6(2):163-8.

62. Grubmüller H, Heller H, Windemuth A, Schulten K. Generalized Verlet algorithm for efficient molecular dynamics simulations with long-range interactions. Molecular Simulation. 1991;6(1-3):121-42.

63. Barnes J, Hut P. A hierarchical O $(\mathrm{N} \log \mathrm{N})$ force-calculation algorithm. nature. 1986;324(6096):446.

64. Hess B, Bekker H, Berendsen HJ, Fraaije JG. LINCS: a linear constraint solver for molecular simulations. Journal of computational chemistry. 1997;18(12):1463-72.

65. Darden T, York D, Pedersen L. Particle mesh Ewald: An N. log (N) method for Ewald sums in large systems. The Journal of chemical physics. 1993;98(12):10089-92.

66. Perler FB. Protein splicing of inteins and hedgehog autoproteolysis: structure, function, and evolution. Cell. 1998;92(1):1-4.

67. Pearson WR. An introduction to sequence similarity ("homology") searching. Current protocols in bioinformatics. 2013;42(1):3.1. -3.1. 8.

68. Albrecht AG, Netz DJ, Miethke M, Pierik AJ, Burghaus O, Peuckert F, et al. SufU is an essential iron-sulfur cluster scaffold protein in Bacillus subtilis. Journal of bacteriology. 2010;192(6):1643-51.

69. Pérard J, de Choudens SO. Iron-sulfur clusters biogenesis by the SUF machinery: close to the molecular mechanism understanding. JBIC Journal of Biological Inorganic Chemistry. 2018;23(4):581-96.

70. Zhang B, Rapolu M, Liang Z, Han Z, Williams PG, Su WW. A dual-intein autoprocessing domain that directs synchronized protein co-expression in both prokaryotes and eukaryotes. Scientific reports. 2015;5:8541.

71. Kirby AJ, Souza BS, Medeiros M, Priebe JP, Manfredi AM, Nome F. Hydroxylamine as an oxygen nucleophile. Chemical evidence from its reaction with a phosphate triester. Chemical Communications. 2008(37):4428-9.

72. Bachmann A-L, Mootz HD. An unprecedented combination of serine and cysteine nucleophiles in a split intein with an atypical split site. Journal of Biological Chemistry. 2015;290(48):28792-804.

73. Simanenko YS, Popov A, Prokop'eva T, Savelova V, Belousova I. Hydroxylamine anionan effective $\alpha$-nucleophile in acyl transfer reactions. Theoretical and Experimental Chemistry. 1994;30(2):61-4.

74. $\mathrm{Xu} \mathrm{M}-\mathrm{Q}$, Perler FB. The mechanism of protein splicing and its modulation by mutation. The EMBO journal. 1996;15(19):5146-53.

75. Volkmann G, Sun W, Liu XQ. Controllable protein cleavages through intein fragment complementation. Protein Science. 2009;18(11):2393-402. 
76. Alliegro MC. Effects of Dithiothreitol on Protein Activity Unrelated to Thiol-Disulfide Exchange: For Consideration in the Analysis of Protein Function with Cleland's Reagent. Analytical biochemistry. 2000;282(1):102-6.

77. Pruitt KD, Tatusova T, Maglott DR. NCBI Reference Sequence (RefSeq): a curated nonredundant sequence database of genomes, transcripts and proteins. Nucleic acids research. 2005;33(suppl_1):D501-D4.

78. Chong S, Shao Y, Paulus H, Benner J, Perler FB, Xu M-Q. Protein Splicing Involving the Saccharomyces cerevisiae VMA Intein The steps in the splicing pathway, side reactions leading to protein cleavage, and establishment of an in vitro splicing system. Journal of Biological Chemistry. 1996;271(36):22159-68.

79. Dearden AK, Callahan B, Van Roey P, Li Z, Kumar U, Belfort M, et al. A conserved threonine spring-loads precursor for intein splicing. Protein Science. 2013;22(5):557-63.

80. Frisch M, Trucks G, Schlegel H, Scuseria G, Robb M, Cheeseman J, et al. Gaussian 03, Rev. C. 02, Gaussian Inc. Wallingford, CT. 2004.

81. Becke AD. Density-functional thermochemistry. III. The role of exact exchange. The Journal of chemical physics. 1993;98(7):5648-52.

82. Burke HM, McSweeney L, Scanlan EM. Exploring chemoselective S-to-N acyl transfer reactions in synthesis and chemical biology. Nature communications. 2017;8(1):1-16.

83. Devaraj NK, Perrin CL. Approach control. Stereoelectronic origin of geometric constraints on N-to-S and N-to-O acyl shifts in peptides. Chemical science. 2018;9(7):1789-94.

84. Monbaliu J-CM, Dive G, Stevens CV, Katritzky AR. Governing parameters of long-range intramolecular S-to-N acyl transfers within (S)-acyl isopeptides. Journal of chemical theory and computation. 2013;9(2):927-34.

85. Kirsch JF, Jencks WP. Base catalysis of imidazole catalysis of ester hydrolysis. Journal of the American Chemical Society. 1964;86(5):833-7.

86. Bak DW, Elliott SJ. Alternative FeS cluster ligands: tuning redox potentials and chemistry. Current opinion in chemical biology. 2014;19:50-8.

(1)

西


bioRxiv preprint doi: https://doi.org/10.1101/2021.07.07.451452; this version posted August 16, 2021. The copyright holder for this preprint (which was not certified by peer review) is the author/funder. All rights reserved. No reuse allowed without permission.

1284

1285

1286

1287

1288

1289

1290 